\title{
A two-step drive-by bridge damage detection using Dual Kalman Filter
}

\author{
Jiantao Li ${ }^{1 \mathrm{a}}$, Xinqun Zhu*a, Siu-seong Law ${ }^{\mathrm{b}}$ and Bijan Samali ${ }^{\mathrm{c}}$ \\ ${ }^{a}$ School of Civil and Environmental Engineering, University of Technology Sydney, Broadway, \\ NSW 2007, Australia. ${ }^{1}$ Email: jiantao.li@student.uts.edu.au \\ *Corresponding author: xinqun.zhu@uts.edu.au \\ ${ }^{b}$ School of Civil Engineering, Chongqing University.Email: siu-seong.law@connect.polyu.hk \\ ${ }^{c}$ School of Computing, Engineering and Mathematics, Western Sydney University, Penrith, NSW \\ 2751, Australia.E-mail: b.samali@westernsydney.edu.au
}

\begin{abstract}
Drive-by bridge inspection using acceleration responses of a passing vehicle has great potential for bridge structural health monitoring. It is, however, known that the road surface roughness is a big challenge for the practical application of this indirect approach. This paper presented a new two-step method for the bridge damage identification from only the dynamic responses of a passing vehicle without the road surface roughness information. A state-space equation of the vehicle model is derived based on Newmark- $\beta$ method. In the first step, the road surface roughness is estimated from the dynamic responses of a passing vehicle using the dual Kalman filter (DKF). In the second step, the bridge damage is identified based on the interaction force sensitivity analysis with Tikhonov regularization. A vehicle-bridge interaction model with a wireless monitoring system has been built in the laboratory. Experimental investigation has been carried out for the interaction force and bridge surface roughness identification. Results show that the proposed method is effective and reliable to identify the interaction force and bridge surface roughness. Numerical simulations have also been conducted to study the effectiveness of the proposed method for bridge damage detection. The vehicle is modelled as a 4-degrees-of-freedom half-car and the bridge is modelled as a simply-supported beam. The local bridge damage is simulated as an elemental flexural stiffness reduction. Effects of measurement noise, surface roughness and vehicle speed on the identification are discussed. The results show that the proposed drive-by inspection strategy is efficient and accurate for a quick review on the bridge conditions.
\end{abstract}

Keywords: drive-by inspection, interaction force sensitivity, Newmark- $\beta$ method, dual Kalman filter, bridge damage detection

\section{Introduction}

The vehicle-bridge interaction (VBI) is crucial to passenger comfort, vehicle safety and decision making on the bridge design and maintenance. Extensive studies have been conducted on the dynamic 
coupling between a bridge and a passing vehicle [1-6]. They have been further extended covering the application to bridge structural health monitoring [7, 8] in recent years. The vehicle responses measured when moving on top of the bridge deck contain dynamic information of the bridge structure. The bridge condition can be predicted from the dynamic response of the passing vehicle.

Indirect bridge health monitoring, also known as the drive-by bridge inspection, was first proposed by Yang et al. [9] to extract the bridge modal frequencies from vehicle responses. The drive-by bridge inspection uses an instrumented moving vehicle to extract the bridge modal parameters and possibly conduct bridge damage detection without instrumentation on the structure. $\mathrm{Bu}$ et al. [10] used the acceleration time histories of a passing vehicle to identify the bridge damage by a sensitivity-based model updating method. The road surface roughness was assumed known, and the flexural stiffness reduction of the bridge deck was identified with good accuracy. The operating displacement of the bridge deck was extracted using a moving device with the bridge curvature serving as the damage index and structural damage can be detected via pre-filtering based on wavelet decomposition [11]. Kong et al. [12] proposed a damage detection method based on the dynamic response transmissibility of a moving vehicle in the VBI system. Two sensors were used to record the dynamic responses of the vehicles when the bridge was subjected to traffic excitation. Damage indicators based on the transmissibility from both the bridge and vehicle responses were also determined and compared. Li and $\mathrm{Au}$ [13] presented a multistage damage detection method based on the modal strain energy and the genetic algorithm. Yang and Yang [14] provided a state-of-the-art review on indirect modal identification and damage detection of the bridge deck with moving test vehicles. More recently, Zhu et al. [15] proposed an approach to identify local bridge anomalies with an instrumented vehicle as a moving sensory system. Interaction forces were obtained from the measured axle and vehicle body accelerations. The interaction forces were found more sensitive to the local damages than acceleration responses from the vehicle. Zhang et al. [16] used the contact-point response of a moving test vehicle for bridge damage detection. All the above studies, the road surface roughness is known or ignored.

The vehicle and bridge systems are coupled at the contact points. It is a typical moving load problem and extensive research has been conducted on the moving force identification techniques to quantify the interaction via measurements from the bridge [17-19]. O'Brien et al. [20] identified the interaction forces from vehicle dynamic responses. The proposed identification algorithm involves formulation of the state space equations of motion of vehicle based on dynamic programming technique, Tikhonov regularisation and the L-curve method. However, the smoothing of the solution by regularization does not yield accurate results on some of the force components. Kalman filter based methods are popular for the joint input-state estimation [21-23]. Lei et al. [24] proposed an algorithm based on sequential application of an extended Kalman estimator and least-squares estimation for the identification of 
state vector of structure and unknown external excitations. Adaptive constrained unscented Kalman filtering (UKF) was proposed for nonlinear system identification in civil engineering [25, 26]. Erazo et al. [27] proposed a Kalman filtering based framework for structural damage assessment under changing environmental conditions. The approach decoupled physical changes caused by structural damage and varying environmental conditions. Aucejo [28] discussed the advantages and limitations of the Kalman-type filtering for solving joint input-state estimation problems with special focus on the applicability of the Augmented Kalman Filter (AKF). A dual Kalman filter (DKF) approach was proposed for estimating the unknown input and states of a linear state-space model by using sparse noisy acceleration measurements [29]. The DKF outperformed the AKF in terms of the quality of the displacement estimates. Liu et al. [30] proposed a state-space model established from the explicit form of Newmark- $\beta$ method which is unconditional stable with properly selected parameters. The algorithm had better performance in force identification compared to conventional state space method with zeroth-order-hold $(\mathrm{ZOH})$ sampling technique [24, 29]. The bridge surface roughness is a critical factor but it is usually unknown in indirect bridge health monitoring. It is also flexible and convenient if non-specialized testing vehicle with less instrumentation can be used to carry out the inspection.

This study adopts the approach in [30] to derive the state-space model of the structure for solving using the DKF and a new two-step approach is proposed to identify the bridge road surface roughness and damage simultaneously from the dynamic response of a passing vehicle. The interaction forces obtained are used for the bridge surface roughness identification and bridge damage detection with a force sensitivity analysis and Tikhonov regularization. The proposed strategy can identify the interaction forces and bridge surface roughness with two vehicle axle responses instead of complete measurements at all degrees-of-freedom (DOFs) of the vehicle. Bridge damage detection can then be conducted with the identified interaction force and surface roughness.

The rest of the paper is outlined as follows. The vehicle-bridge interaction model is introduced first. The state space representation of the vehicle model by Newmark- $\beta$ method is derived followed by the introduction of the DKF to solve the state space model. The bridge damage detection with the force sensitivity analysis is then introduced. Numerical and experimental investigations are then carried out for the force identification and bridge deck surface roughness estimation. Study on the bridge damage detection will follow with conclusions drawn for the study.

\section{Vehicle-bridge interaction model}

The half-car model of vehicle shown in Figure 1 consists of 4 DOFs. It moves over a simply-supported bridge with road surface roughness at a constant speed $v$. 


\subsection{Equation of motion of the bridge}

The supporting bridge is discretised into $N$ Euler-Bernoulli beam finite elements. The elemental mass and stiffness matrices are obtained using Hermitian cubic interpolation shape functions. The equation of motion of the bridge structure can be written as:

$$
\mathbf{M}_{b} \ddot{\mathbf{d}}_{b}+\mathbf{C}_{b} \dot{\mathbf{d}}_{b}+\mathbf{K}_{b} \mathbf{d}_{b}=\mathbf{H}_{c} \mathbf{P}_{\text {int }}
$$

where $\mathbf{M}_{b}, \mathbf{C}_{b}, \mathbf{K}_{b}$ are the mass, damping and stiffness matrices of the bridge, respectively; Rayleigh damping is assumed with $\mathbf{C}_{\mathrm{b}}=\alpha_{1} \mathbf{M}_{\mathrm{b}}+\alpha_{2} \mathbf{K}_{\mathrm{b}}$ and $\alpha_{1}$ and $\alpha_{2}$ are constants of proportionality. $\mathbf{P}_{\text {int }}=$ $\left\{P_{1}(t), P_{2}(t)\right\}^{T}$ is the vehicle-bridge interaction force vector. $\mathbf{d}_{b}, \dot{\mathbf{d}}_{b}, \ddot{\mathbf{d}}_{b}$ are the vectors of displacement, velocity and acceleration responses of the bridge respectively. $\mathbf{H}_{c} \mathbf{P}_{\text {int }}$ is the equivalent nodal load vector from the bridge-vehicle interaction force with

$$
\mathbf{H}_{c}=\left\{\begin{array}{lllllll}
0 & \ldots & 0 & \ldots & \boldsymbol{H}_{1} & \ldots & 0 \\
0 & \ldots & \boldsymbol{H}_{2} & \ldots & 0 & \ldots & 0
\end{array}\right\}^{T} \in R^{N N \times 2}
$$

where $\mathbf{H}_{c}$ is a matrix with null entries at the DOFs corresponding to the nodes of the beam elements on which the loads are acting. $N N$ is the total number of DOFs of the bridge structure after considering the boundary conditions. The components of vector $H_{i}(i=1,2)$ evaluated for the ith interactive force on the $j$ th finite element can be written in global coordinates as:

$$
\boldsymbol{H}_{i}=\left\{\begin{array}{c}
1-3\left(\frac{\hat{x}_{i}(t)-(j-1) l}{l}\right)^{2}+2\left(\frac{\hat{x}_{i}(t)-(j-1) l}{l}\right)^{3} \\
\left(\hat{x}_{i}(t)-(j-1) l\right)\left(\frac{\hat{x}_{i}(t)-(j-1) l}{l}-1\right)^{2} \\
3\left(\frac{\hat{x}_{i}(t)-(j-1) l}{l}\right)^{2}-2\left(\frac{\hat{x}_{i}(t)-(j-1) l}{l}\right)^{3} \\
\left(\hat{x}_{i}(t)-(j-1) l\right)\left(\left(\frac{\hat{x}_{i}(t)-(j-1) l}{l}\right)^{2}-\left(\frac{\hat{x}_{i}(t)-(j-1) l}{l}\right)\right)
\end{array}\right\}
$$

with $(j-1) l \leq \hat{x}_{i}(t) \leq j l$ and $l$ is the length of finite element.

\subsection{Equation of motion for the vehicle model}

The equation of motion of the vehicle can be written as follows

$$
\left[\begin{array}{cc}
\mathbf{M}_{v 1} & 0 \\
0 & \mathbf{M}_{v 2}
\end{array}\right] \ddot{\mathbf{X}}_{v}+\left[\begin{array}{ll}
\mathbf{C}_{v 11} & \mathbf{C}_{v 12} \\
\mathbf{C}_{v 21} & \mathbf{C}_{v 22}
\end{array}\right] \dot{\mathbf{X}}_{v}+\left[\begin{array}{ll}
\mathbf{K}_{v 11} & \mathbf{K}_{v 12} \\
\mathbf{K}_{v 21} & \mathbf{K}_{v 22}
\end{array}\right] \mathbf{X}_{v}=-\left\{\begin{array}{c}
0 \\
\mathbf{P}_{\text {int }}
\end{array}\right\}+\left\{\begin{array}{c}
0 \\
\mathbf{M}_{s}
\end{array}\right\}
$$

where $\mathbf{X}_{v}=\left\{y_{v}, \theta_{v}, y_{1}, y_{2}\right\}^{T}$ is the response vector of the vehicle. $\mathbf{M}_{v 1}, \mathbf{M}_{v 2}, \mathbf{C}_{v 11}, \mathbf{C}_{v 12}, \mathbf{C}_{v 21}, \mathbf{C}_{v 22}$, $\mathbf{K}_{v 11}, \mathbf{K}_{v 12}, \mathbf{K}_{v 21}, \mathbf{K}_{v 22}$ are the mass, damping and stiffness sub-matrices of the vehicle model respectively. $\mathbf{M}_{s}=\left\{M_{s 1}, M_{s 2}\right\}^{T}$ is the static load vector of the vehicle. 
Combining Eqs. (1) and (4), the equation of motion of the coupled vehicle-bridge system can be written as:

$$
\mathbf{M}_{g} \ddot{\mathbf{u}}+\mathbf{C}_{g} \dot{\mathbf{u}}+\mathbf{K}_{g} \mathbf{u}=\mathbf{P}_{g}
$$

where $\mathbf{u}$ is the response vector of coupled system. $\mathbf{M}_{g}, \mathbf{C}_{g}$ and $\mathbf{K}_{g}$ are the combined system mass, damping and stiffness matrices respectively, $\mathbf{P}_{\boldsymbol{g}}$ is the force vector. The matrices in Eqs. (4) and (5) are given in Appendix I. When the vehicle and bridge parameters and the surface roughness are known, the dynamic responses of the system can be calculated at each time step using Newmark- $\beta$ method.

\subsection{Road surface roughness}

The road surface roughness in time domain can be simulated from [31]

$$
r(x)=\sum_{i=1}^{N_{f}} \sqrt{4 S_{d}\left(f_{i}\right) \Delta f} \cos \left(2 \pi f_{i} x+\theta_{i}\right)
$$

where $S_{d}(f)$ is the displacement power spectral density of the road surface roughness, $f_{i}=i \Delta f$ is

the spatial frequency(cycles/m), $\Delta f=\frac{1}{N_{f} \Delta}, \Delta$ is the distance interval between successive ordinates of the surface profile, $N_{f}$ is the number of data points, and $\theta_{i}$ is a set of independent random phase angle uniformly distributed between 0 and $2 \pi$. Class A, B and C road surface roughness from ISO (8606) [32] are used in the simulation.

\section{Estimation of the state vector and unknown input forces of the vehicle by DKF}

The DKF is used to identify the vehicle state vector and the interaction forces between the vehicle and bridge. Analytical recursive solutions are derived in this section with the Newmark- $\beta$ method from the equation of motion of the vehicle system. When the interaction forces between the vehicle and bridge are identified from the vehicle acceleration responses, the displacement responses of the bridge at the contact points can be calculated by introducing the interaction forces as input to the bridge system (Eq. (1)). With the contact point responses and vehicle responses, the roughness can be calculated from the interaction forces. The power spectrum density (PSD) of the calculated bridge surface roughness can be obtained.

\subsection{State space model of the vehicle}


In order to simplify, the subscript " $v$ " is removed, and $\mathbf{x}, \dot{\mathbf{x}}$ and $\ddot{\mathbf{x}}$ are respectively the vehicle displacement, velocity and acceleration responses. Eq. (4) can be rewritten as

$$
\mathbf{M}_{v} \ddot{\mathbf{x}}(\mathrm{t})+\mathbf{C}_{v} \dot{\mathbf{x}}(\mathrm{t})+\mathbf{K}_{v} \mathbf{x}(\mathrm{t})=\mathbf{L P}(\mathrm{t})
$$

where $\mathbf{L}=[00 ; 00 ; 10 ; 01]$ is the influence matrix associated with the unknown excitation vector $\mathbf{P}(\mathrm{t})=\mathbf{M}_{s}-\mathbf{P}_{\text {int }}$ on the vehicle system. $\quad \mathbf{M}_{v}=\left[\begin{array}{cc}\mathbf{M}_{v 1} & 0 \\ 0 & \mathbf{M}_{v 2}\end{array}\right], \quad \mathbf{C}_{v}=\left[\begin{array}{ll}\mathbf{C}_{v 11} & \mathbf{C}_{v 12} \\ \mathbf{C}_{v 21} & \mathbf{C}_{v 22}\end{array}\right], \quad \mathbf{K}_{v}=$ $\left[\begin{array}{ll}\mathbf{K}_{v 11} & \mathbf{K}_{v 12} \\ \mathbf{K}_{v 21} & \mathbf{K}_{v 22}\end{array}\right] . \mathbf{P}(\mathrm{t})$ is the dynamic force vector acting at the contact points between the vehicle and bridge deck.

The explicit Newmark- $\beta$ method [30] is based on an assumed variation of acceleration between two time instants as

$$
\begin{aligned}
& \ddot{\mathbf{x}}=(1-\gamma) \ddot{\mathbf{x}}_{i}+\gamma \ddot{\mathbf{x}}_{i+1} \quad(0 \leq \gamma \leq 1) \\
& \ddot{\mathbf{x}}=(1-2 \beta) \ddot{\mathbf{x}}_{i}+2 \beta \ddot{\mathbf{x}}_{i+1} \quad(0 \leq \beta \leq 0.5)
\end{aligned}
$$

Integrating the acceleration between $t_{i}$ and $t_{i+1}$, the displacement and velocity at $t_{i+1}$ can be obtained as

$$
\begin{gathered}
\dot{\mathbf{x}}_{i+1}=\dot{\mathbf{x}}_{i}+\Delta t \ddot{\mathbf{x}} \\
\mathbf{x}_{i+1}=\mathbf{x}_{i}+\Delta t \dot{\mathbf{x}}_{i}+\frac{1}{2} \Delta t^{2} \ddot{\mathbf{x}}
\end{gathered}
$$

The iterative form of the explicit Newmark- $\beta$ method is obtained as [28]

$$
\left[\begin{array}{c}
\mathbf{x}_{i+1} \\
\dot{\mathbf{x}}_{i+1} \\
\ddot{\mathbf{x}}_{i+1}
\end{array}\right]=\left[\begin{array}{c}
\mathbf{A}_{0} \\
\mathbf{B}_{0} \\
\mathbf{C}_{0}
\end{array}\right] \mathbf{L P}_{i+1}+\left[\begin{array}{lll}
\mathbf{A}_{d} & \mathbf{A}_{v} & \mathbf{A}_{a} \\
\mathbf{B}_{d} & \mathbf{B}_{v} & \mathbf{B}_{a} \\
\mathbf{C}_{d} & \mathbf{C}_{v} & \mathbf{C}_{a}
\end{array}\right]\left[\begin{array}{c}
\mathbf{x}_{i} \\
\dot{\mathbf{x}}_{i} \\
\ddot{\mathbf{x}}_{i}
\end{array}\right]
$$

where $\mathbf{A}_{0}=\left(\mathbf{K}+\frac{1}{\beta \Delta t^{2}} \mathbf{M}+\frac{\gamma}{\beta \Delta t} \mathbf{C}\right)^{-1}, \mathbf{A}_{d}=\mathbf{A}_{0}\left(\frac{1}{\beta \Delta t^{2}} \mathbf{M}+\frac{\gamma}{\beta \Delta t} \mathbf{C}\right), \mathbf{A}_{v}=\mathbf{A}_{0}\left[\frac{1}{\beta \Delta t} \mathbf{M}+\left(\frac{\gamma}{\beta}-1\right) \mathbf{C}\right]$,

$\mathbf{A}_{a}=\mathbf{A}_{0}\left[\left(\frac{1}{2 \beta}-1\right) \mathbf{M}+\frac{\Delta t}{2}\left(\frac{\gamma}{\beta}-2\right) \mathbf{C}\right], \mathbf{C}_{0}=\frac{1}{\beta \Delta t^{2}} \mathbf{A}_{0}, \mathbf{C}_{d}=\frac{-1}{\beta \Delta t^{2}} \mathbf{A}_{0} \mathbf{K}, \mathbf{C}_{v}=\frac{-1}{\beta \Delta t^{2}} \mathbf{A}_{0}(\mathbf{C}+\Delta t \mathbf{K})$,

$\mathbf{C}_{a}=\frac{1}{\beta \Delta t^{2}} \mathbf{A}_{0}\left[(\gamma-1) \Delta t \mathbf{C}-\beta \Delta t^{2}\left(\frac{1}{2 \beta}-1\right) \mathbf{K}\right], \mathbf{B}_{0}=\frac{\gamma}{\beta \Delta t} \mathbf{A}_{0}, \mathbf{B}_{d}=\frac{-\gamma}{\beta \Delta t} \mathbf{A}_{0} \mathbf{K}$,

$\mathbf{B}_{v}=\frac{\gamma}{\beta \Delta t} \mathbf{A}_{0}\left[\left(\frac{\beta \Delta t}{\gamma}-\Delta t\right) \mathbf{K}+\frac{1}{\gamma \Delta t} \mathbf{M}\right], \mathbf{B}_{a}=\frac{-\gamma}{\beta \Delta t} \mathbf{A}_{0}\left[\left(\frac{\beta \Delta t^{2}}{\gamma}-\frac{\Delta t^{2}}{2}\right) \mathbf{K}+\left(\frac{1}{\gamma}-1\right) \mathbf{M}\right]$.

For a state vector $\mathbf{X}_{i}=\left[\mathbf{x}_{i}^{T} \dot{\mathbf{x}}_{i}^{T} \ddot{\mathbf{x}}_{i}^{T}\right]^{T}$, Eq. (12) can be rewritten in matrix form as

$$
\mathbf{X}_{i+1}=\mathbf{A} \mathbf{X}_{i}+\mathbf{B} \mathbf{P}_{i+1}
$$


where

$$
\mathbf{A}=\left[\begin{array}{lll}
\mathbf{A}_{d} & \mathbf{A}_{v} & \mathbf{A}_{a} \\
\mathbf{B}_{d} & \mathbf{B}_{v} & \mathbf{B}_{a} \\
\mathbf{C}_{d} & \mathbf{C}_{v} & \mathbf{C}_{a}
\end{array}\right], \quad \mathbf{B}=\left[\begin{array}{c}
\mathbf{A}_{0} \\
\mathbf{B}_{0} \\
\mathbf{C}_{0}
\end{array}\right] \mathbf{L}
$$

\subsubsection{The output equation}

Vector $\boldsymbol{y} \in R^{n s \times 1}$ represents the output of the structural system and it can be assembled from measurements with

$$
\mathbf{y}=\mathbf{R}_{a} \ddot{\mathbf{x}}+\mathbf{R}_{v} \dot{\mathbf{x}}+\mathbf{R}_{b} \mathbf{x}
$$

where $\mathbf{R}_{a}, \mathbf{R}_{v}$ and $\mathbf{R}_{b} \in R^{n s \times N N}$ are the output influence matrices for the measured acceleration, velocity and displacement, respectively, $n s$ is the dimension of the measured responses and $N N$ is the number of DOFs of the structure.

Letting $\mathbf{R}=\left[\mathbf{R}_{d} \mathbf{R}_{v} \mathbf{R}_{a}\right.$ ], Eq.(14) can be rewritten into the following discrete form as

$$
\mathbf{y}_{i}=\mathbf{R} \mathbf{X}_{i}
$$

\subsubsection{State-space model}

The state space representation of the vehicle-bridge dynamic system can be obtained from Eqs. (13) and (15) as

$$
\left\{\begin{array}{l}
\mathbf{X}_{i+1}=\mathbf{A X}_{i}+\mathbf{B} \mathbf{P}_{i+1}+\boldsymbol{v}_{i}^{x} \\
\mathbf{y}_{i}=\mathbf{R} \mathbf{X}_{i}+\boldsymbol{w}_{i}
\end{array}\right.
$$

where $\boldsymbol{v}_{\boldsymbol{i}}^{\boldsymbol{x}}$ and $\boldsymbol{w}_{i}$ are the process noise and the measurement noise vectors respectively representing uncertainties in the modelling and the measurement processes respectively. The noise vectors $\boldsymbol{v}_{\boldsymbol{i}}^{\boldsymbol{x}}$ and $\boldsymbol{w}_{i}$ are assumed Gaussian white with covariance $\boldsymbol{Q}^{\boldsymbol{x}}$ and $\boldsymbol{Q}^{\boldsymbol{y}}$ respectively.

The state space formulation in Eq. (16) is not in standard form, as the information of input vector at time $i+1$ is required in the state equation. A reduced state $\overline{\mathbf{X}}_{i}=\mathbf{X}_{i}-\mathbf{B} \mathbf{P}_{i}$ can be further formulated by transforming Eq. (16) as

$$
\left\{\begin{array}{l}
\overline{\mathbf{X}}_{i+1}=\mathbf{A} \overline{\mathbf{X}}_{i}+\mathbf{A B P}_{i}+\boldsymbol{v}_{i}^{x} \\
\mathbf{y}_{i}=\mathbf{R} \overline{\mathbf{X}}_{i}+\mathbf{R B} \mathbf{P}_{i}+\boldsymbol{w}_{i}
\end{array}\right.
$$




\subsection{Dual Kalman Filter}

Joint input-state estimation is conducted in this section. A random walk model is introduced to represent the state equation for the input $\mathbf{P}_{i}$ as

$$
\mathbf{P}_{i+1}=\mathbf{P}_{i}+v_{i}^{p}
$$

where $\boldsymbol{v}_{\boldsymbol{i}}^{\boldsymbol{p}}$ is a zero mean Gaussian white process with covariance matrix $\boldsymbol{Q}^{\boldsymbol{p}}$ that is determined by Eq. (20). Combining Eqs. (17b) and (18) gives a new state-space equation for the input as

$$
\left\{\begin{array}{l}
\mathbf{P}_{i+1}=\mathbf{P}_{i}+v_{i}^{p} \\
\mathbf{y}_{i}=\mathbf{R B P}_{i}+\mathbf{R} \overline{\mathbf{X}}_{i}+\boldsymbol{w}_{i}
\end{array}\right.
$$

where the observation is $\mathbf{y}_{i}$, and the new state is $\mathbf{P}_{i}$. A sequential implementation of the Kalman Filter described in Eqs. (17) and (19) can give the state $\mathbf{X}_{i}$ and input force $\mathbf{P}_{i}$. This procedure was named as Dual Kalman filter (DKF) [29] with the following general description.

1. Initialization of the state and input force at $t_{0}$

Estimation of the initial state $\overline{\mathbf{X}}_{0}$ and input force value $\widehat{\mathbf{P}}_{0}$ and their corresponding covariance matrices $\mathbf{G}_{\mathbf{0}}^{\boldsymbol{x}}$ and $\mathbf{G}_{\mathbf{0}}^{\boldsymbol{p}}$

2. For each time instant $\mathrm{t}_{i}\left(i=1, \ldots, N_{t}\right)$

2.1 Prediction stage for the input

Evolution of the input and prediction of covariance input with

$$
\mathbf{P}_{i}^{-}=\mathbf{P}_{i-1} ; \quad \mathbf{G}_{i}^{p-}=\mathbf{G}_{i-1}^{p}+Q^{p}
$$

2.2 Update stage for the input

Kalman gain for input with

$$
\mathbf{K}_{i}^{p}=\mathbf{G}_{i}^{p-} \mathbf{J}^{T}\left(\mathrm{JG}_{i}^{p-} \mathbf{J}^{T}+Q^{y}\right)^{-1}
$$

where $\mathbf{J}=\mathbf{R B}$

Improved predictions of input with

$$
\widehat{\mathbf{P}}_{i}=\mathbf{P}_{i}^{-}+\mathbf{K}_{i}^{p}\left(\mathbf{y}_{i}-\mathbf{J} \mathbf{P}_{i}-\mathbf{R} \overline{\mathbf{X}}_{i-1}\right) ; \quad \mathbf{G}_{i}^{p}=\mathbf{G}_{i}^{p-}-\mathbf{K}_{i}^{p} \mathbf{J G}_{i}^{p-}
$$

2.3 Prediction stage for the state:

Evolution of state and prediction of covariance of state with

$$
\overline{\mathbf{X}}_{\boldsymbol{i}}^{-}=\mathbf{A} \overline{\mathbf{X}}_{i-1}+\mathbf{A B} \widehat{\mathbf{P}}_{i} ; \quad \mathbf{G}_{i}^{x-}=\mathbf{A G}_{i-1}^{x} \mathbf{A}+Q^{x}
$$

2.4 Update stage for the state

Kalman gain for state -

$$
\mathbf{K}_{i}^{x}=\mathbf{G}_{i}^{x-} \mathbf{R}^{T}\left(\mathbf{R G}_{i}^{x-} \mathbf{R}^{T}+Q^{y}\right)^{-1}
$$

Improved predictions of state - 


$$
\overline{\mathbf{X}}_{i}=\overline{\mathbf{X}}_{i}^{-}+\mathbf{K}_{i}^{x}\left(\mathbf{y}_{i}-\mathbf{R} \overline{\mathbf{X}}_{i}^{-}-\mathbf{J} \widehat{\mathbf{P}}_{i}\right) ; \quad \mathbf{G}_{i}^{x}=\mathbf{G}_{i}^{x-}-\mathbf{K}_{i}^{x} \mathbf{R G}_{i}^{x-}
$$

It is worth noting that the procedure needs a priori information on the expected value and covariance of the state and input at time $t_{0}$. The value of the process noise $\boldsymbol{Q}^{\boldsymbol{p}}$ in Eq. (18) must be properly chosen so that an accurate estimate of the unobserved state and the unknown input could be estimated. The process noise covariance matrices $\boldsymbol{Q}^{\boldsymbol{x}}$ and $\boldsymbol{Q}^{\boldsymbol{p}}$ represent the suitability of the formulated model of the system. The observation noise covariance $\boldsymbol{Q}^{y}$ represents the accuracy of the acquired measurements. The vehicle model is assumed accurate and the process noise for the vehicle state is set to a small value of $\boldsymbol{Q}^{\boldsymbol{x}}=10^{-20} \times \mathbf{I}$ where $\mathbf{I}$ is an identity matrix with dimension equals to 4 corresponding to the number of DOFs of the vehicle system. The initial values of the covariance of the state are taken the same as for the process noise. Since the vehicle system is assumed at rest at the beginning of simulation, the expected values for the initial conditions are all assumed null.

The expected value and covariance of the input force are also needed. $\boldsymbol{Q}^{\boldsymbol{p}}$ is a tuning parameter to smooth the variation of the time history of the input. Its value significantly affects the quality of the estimated solutions [29] and it is determined as described below.

The current state update is directly related to the innovation $\mathbf{I}_{\text {nov }}^{\boldsymbol{i}}=\mathbf{y}_{i}-\mathbf{R} \overline{\mathbf{X}}_{\boldsymbol{i}}^{-}-\mathbf{J} \widehat{\mathbf{P}}_{\boldsymbol{i}}$ from the Kalman filtering algorithm presented above. The innovation can be seen either as the prediction error of the state given the measurements or as a measure of the information brought by the new measurement. The optimal value $\widehat{\boldsymbol{Q}}^{p}$ can be determined by minimising the innovation norm as [28, 29],

$$
\widehat{\boldsymbol{Q}}^{\boldsymbol{p}}=\underset{\boldsymbol{Q}^{\boldsymbol{p}}}{\operatorname{argmin}} \sum_{i=1}^{N_{t}}\left\|\mathbf{y}_{i}-\mathbf{R} \overline{\mathbf{X}}_{\boldsymbol{i}}^{-}-\mathbf{J P}_{\boldsymbol{i}}\right\|_{2}^{2} / N_{t}
$$

where $N_{t}$ is the number of time steps.

\section{Damage identification via the interaction forces}

The damage index $\alpha_{s}^{j}$ is defined as a fractional change in the $j$ th elemental flexural stiffness of the bridge deck, and the damaged parameter is defined as

$$
(E I)_{d}^{j}=(E I)_{0}^{j}\left(1-\alpha_{s}^{j}\right) \quad\left(0 \leq \alpha_{s}^{j} \leq 1, j=1,2, \ldots m\right)
$$

where $(E I)_{0}^{j}$ is the parameter of the $j$ th element when the bridge is intact.

Taking the first derivative of Eq. (5) with respect to the damage index $\alpha_{s}^{j}$ of the bridge to have 


$$
M_{g} \ddot{\boldsymbol{S}}+C_{g} \dot{\boldsymbol{S}}+K_{g} \boldsymbol{S}=\overline{\boldsymbol{P}}
$$

where $\ddot{\boldsymbol{S}}=\left[\frac{\partial \ddot{\mathbf{d}}_{b}}{\partial\left(\alpha_{s}^{j}\right)} ; \frac{\partial \ddot{\mathbf{x}}_{v}}{\partial\left(\alpha_{s}^{j}\right)}\right], \dot{\boldsymbol{S}}=\left[\frac{\partial \dot{\mathbf{d}}_{b}}{\partial\left(\alpha_{s}^{j}\right)} ; \frac{\partial \dot{\mathbf{x}}_{v}}{\partial\left(\alpha_{s}^{j}\right)}\right], \boldsymbol{S}=\left[\frac{\partial \mathbf{d}_{b}}{\partial\left(\alpha_{s}^{j}\right)} ; \frac{\partial \mathbf{x}_{v}}{\partial\left(\alpha_{s}^{j}\right)}\right]$ are the response sensitivity matrices with respect to the damage index $\alpha_{s}^{j} ; \overline{\boldsymbol{P}}=\left[\begin{array}{c}\frac{-\partial K_{b}}{\partial\left(\alpha_{s}^{j}\right)} \mathbf{d}_{b}-\alpha_{2} \frac{-\partial K_{b}}{\partial\left(\alpha_{s}^{j}\right)} \dot{\mathbf{d}}_{b} \\ \mathbf{0}\end{array}\right]$. They can be obtained by solving Eq.(22) using Newmark integration method.

The sensitivity of the interaction forces $\boldsymbol{S}_{f}$ can be derived from Eq. (7) as the first partial derivative with respect to the stiffness parameters as

$$
\boldsymbol{S}_{f}=\frac{\partial \mathbf{P}}{\partial\left(\alpha_{s}^{j}\right)}=\left(\mathbf{L}^{T} \mathbf{L}\right)^{-1} \mathbf{L}^{T}\left(\mathbf{M}_{v} \frac{\partial \ddot{\mathbf{x}}_{v}}{\partial\left(\alpha_{s}^{j}\right)}+\mathbf{C}_{v} \frac{\partial \dot{\mathbf{x}}_{v}}{\partial\left(\alpha_{s}^{j}\right)}+\mathbf{K}_{v} \frac{\partial \mathbf{x}_{v}}{\partial\left(\alpha_{s}^{j}\right)}\right)
$$

Zero initial values for the vector of damage index are assumed as $\boldsymbol{\theta}_{0}=\left\{\alpha_{s}^{1}, \alpha_{s}^{2}, \ldots, \alpha_{s}^{m}\right\}^{T}=\mathbf{0}$. The difference between the measured and the analytical interaction forces then becomes

$$
\Delta \mathbf{P}_{r}=\mathbf{P}_{i n t}^{m}-\mathbf{P}_{r} \quad(r=0,1,2, \ldots)
$$

where subscript $r$ denotes the value at the $r$ th iteration and $\mathbf{P}_{r}$ is the $r$ th iterative analytical interaction forces. Quantities with subscript $r=0$ refer to the set of initial values. $\mathbf{P}_{\text {int }}^{m}$ is the identified interaction forces in the first step. The vector of flexural stiffness change, $\Delta \boldsymbol{\theta}_{r}$, can be solved from the following sensitivity equation as:

$$
\boldsymbol{S}_{f, r} \Delta \boldsymbol{\theta}_{r}=\Delta \mathbf{P}_{r} \quad(r=0,1,2, \ldots)
$$

The updated damage index vector $\boldsymbol{\theta}_{r+1}=\boldsymbol{\theta}_{r}+\Delta \boldsymbol{\theta}_{r}$ is calculated in the next iteration followed by the calculation on the interaction forces and sensitivity. The above process is repeated until the identified damage index increment in the successive iteration is smaller than a predefined tolerance level.

$$
\left\|\Delta \boldsymbol{\theta}_{r}\right\|<\text { tolerance }
$$

where $\|\cdot\|$ is the norm of the matrix.

The set of damage index increments $\Delta \boldsymbol{\theta}_{r}$ obtained from Eq. (25) using a straight forward leastsquares method would be unbound. Tikhonov regularization is used for optimizing the following objective function as

$$
\mathrm{J}\left(\Delta \boldsymbol{\theta}_{r}, \lambda_{p}^{r}\right)=\left\|\boldsymbol{S}_{d, r} \Delta \boldsymbol{\theta}_{r}-\Delta \mathbf{P}_{r}\right\|^{2}+\lambda_{p}^{r}\left\|\Delta \boldsymbol{\theta}_{r}\right\|
$$

The optimal value of regularization parameter $\lambda_{p}^{r}$ is determined using L-curve method [33]. A flow chart that shows the proposed two-stage drive-by bridge damage detection method is given in Figure 
2. In the practice, the bridge condition is unknown. The bridge road surface roughness and damage can be identified simultaneously through an iterative procedure. The bridge damage is identified using the predicted road surface roughness with the intact bridge model and then the identified bridge model can be used to update the road surface roughness. The procedure can be repeated until both the changes of bridge damage road surface roughness reach the given tolerances.

\section{Studies on interaction force identification and surface roughness estimation}

\section{$5.1 \quad$ Numerical example}

Properties of the target bridge model studied are: $L=30 \mathrm{~m}, \rho A=6.0 \times 10^{3} \mathrm{~kg} / \mathrm{m}, E I=2.5 \times$ $10^{10} \mathrm{Nm}^{2}$. The coefficients for Rayleigh damping are $\alpha_{1}=0.343$ and $\alpha_{2}=0.001$. Parameters of the vehicle are: $m_{v}=17735 \mathrm{~kg}, I_{v}=1.47 \times 10^{5} \mathrm{kgm}^{2}, S=4.2 \mathrm{~m}, a 1=0.519, a 2=0.481, m_{1}=$ $1500 \mathrm{~kg}, k_{s 1}=2.47 \times 10^{6} \mathrm{~N} / \mathrm{m}, k_{t 1}=3.74 \times 10^{6} \mathrm{~N} / \mathrm{m}, c_{s 1}=3.00 \times 10^{4} \mathrm{~N} / \mathrm{m} / \mathrm{s}, c_{t 1}=0.00 \mathrm{~N} /$ $\mathrm{m} / \mathrm{s}, m_{2}=1000 \mathrm{~kg}, k_{s 2}=4.23 \times 10^{6} \mathrm{~N} / \mathrm{m}, k_{t 2}=4.60 \times 10^{6} \mathrm{~N} / \mathrm{m}, c_{s 2}=4.00 \times 10^{4} \mathrm{~N} / \mathrm{m} /$ $s, c_{t 2}=0.00 \mathrm{~N} / \mathrm{m} / \mathrm{s}$. The bridge deck is represented by ten finite elements and acceleration responses of the moving vehicle axles are calculated as the measured signals with sampling frequency of $1000 \mathrm{~Hz}$. All these parameters will be adopted for the following studies unless otherwise stated.

\subsubsection{Force identification and surface roughness estimation}

Figure 3 shows the vehicle axle responses when it is moving at $20 \mathrm{~m} / \mathrm{s}$ on top of the bridge deck with Class B surface roughness. The proposed method is applied for the identification of the axle interaction forces. Eq. (2) is used to determine the value of $\boldsymbol{Q}^{\boldsymbol{p}}$. Figure 4 shows the innovation norm obtained using the acceleration time histories of the vehicle axles as listed in Eq. (20). The optimal value of $\boldsymbol{Q}^{p}=5 \times 10^{23}$ is chosen which corresponds to the minimum mean error norm.

A relative percentage error (RPE) is used to evaluate the identified results as

$$
R P E=\frac{\left\|\boldsymbol{P}_{\text {est }}-\boldsymbol{P}_{\text {true }}\right\|}{\left\|\boldsymbol{P}_{\text {true }}\right\|} \times 100 \%
$$

where $\boldsymbol{P}_{\text {est }}$ and $\boldsymbol{P}_{\text {true }}$ are the estimated and the true time series respectively; $\|\cdot\|$ is the norm of vector. Figures 5(a) and 5(b) show the identified interaction forces and the road surface roughness, respectively. They are noted matching the true curves well. It could be concluded that the proposed strategy is able to identify the interaction forces and bridge surface roughness with high accuracy when there is no measurement noise.

The effect of measurement noise is studied next. White noise is added to the calculated acceleration responses to simulate the polluted measurement as, 


$$
a c c_{m}=a c c_{c a l}+E_{p} * N_{o i s e} * \sigma\left(a c c_{c a l}\right)
$$

where $a c c_{c a l}$ is the calculated acceleration response; $E_{p}$ is the noise level; $N_{\text {oise }}$ is a standard normal distribution vector with zero mean and unit variance; and $\sigma\left(a c c_{c a l}\right)$ is the standard deviation of the calculated acceleration response. $2 \%, 5 \%$ and 10\% random noise are added to the responses separately when the vehicle moves over a Class B surface roughness at a speed of $20 \mathrm{~m} / \mathrm{s}$. Figure 6(a) presents the identified interaction forces for the case with 5\% measurement noise. Figures 6(b) and 6(c) show the identified bridge surface roughness and its PSD respectively. The RPE for the identified results are given in Table 1. The identified surface roughness is noted deviating from the true values. The PSDs of the identified road profile in Figure 6(c) shows that the discrepancies mainly exist at high frequency. The measurement noise is noted to have large effect on the identification results. It could be concluded that when the level of measurement noise is low, i.e. not larger than $5 \%$, the identified results are acceptable.

\subsubsection{Effect of moving speed and sampling frequency}

The effects of vehicle moving speed at $10 \mathrm{~m} / \mathrm{s}$ and $30 \mathrm{~m} / \mathrm{s}$ on deck with Class B road surface roughness are studied. Measurement noise of $5 \%$ is considered. Sampling frequency $\mathrm{f}_{\mathrm{s}}=1000 \mathrm{~Hz}$ and $5000 \mathrm{~Hz}$ are used. The RPE for the identified results are tabulated in Table 2. Figure 7 shows the identified interaction forces and bridge surface roughness when $\mathrm{f}_{\mathrm{s}}=1000 \mathrm{~Hz}$ for different vehicle speeds. Results from both Table 2 and Figure 7(b) show that a high moving speed will reduce the deviation of the identified surface roughness with improved accuracy. As shown in Figure 7(a), the interaction forces can be identified accurately for all vehicle speeds. The identified road roughness has a large accumulation error with the long duration due to the low vehicle speed. Figure 8 shows the identified results with different sampling frequencies, $1000 \mathrm{~Hz}$ and $5000 \mathrm{~Hz}$. Figure $8(\mathrm{a})$ shows that a high sampling frequency will reduce the RPE in the force identification. An inspection of Figure 8(a) also shows that the identified force values at the peaks and valleys using a higher sampling frequency are more accurate than those by using a lower one. As Shown in Figure 8(b), the identified errors for the road roughness are also reduced with the high sampling rate. The above results demonstrates that a high vehicle speed would be beneficial for a quick pavement surface inspection to reduce the traffic interruption.

\subsection{Experimental investigation}


A vehicle-bridge interaction model was built in the laboratory. The bridge model shown in Figure 9(a) consists of three rectangular steel beams with width and depth of $100 \mathrm{~mm}$ and $15 \mathrm{~mm}$, respectively. The main beam in the middle is a two equal span continuous simply-supported bridge model with a span length of $3 \mathrm{~m}$. A leading and trailing beam are placed before and after the main beam to allow for acceleration and deceleration of the vehicle and the length of these beams is $3 m$. An U-shaped aluminium section is glued along the centre of the upper surface of the beam as a direction guide for the vehicle. A model vehicle is pulled along the guide with an electric motor. The two-axle vehicle model was fabricated as shown in Figure 9(b). The wheel of the vehicle model is made of plastic rubber with very light mass and very large stiffness. The masses $m_{1}, m_{2}$ for the front and rear axles are very small and ignored in this study. Eq. (7) can be simplified as a 2-DOF system and the right hand side of the equation will be the dynamic interaction force between the vehicle and bridge. The stiffness and damping of the tyres $K_{t}, C_{t}$ are not needed. The spring is used as the suspension system in the vehicle model, and its damping $C_{S}$ is not considered in this study. The mass and rotational inertia of the vehicle body were $m_{v}=4.9 \mathrm{~kg}$ and $I_{v}=0.06 \mathrm{kgm}^{2}$ respectively. The stiffness of the vehicle suspension system were $k_{s 1}=k_{s 2}=1.3 \times 10^{5} \mathrm{~N} / \mathrm{m}$. These parameters were determined by modal testing of the vehicle.

A wireless sensory system was also setup for the test with devices manufactured by Beanair Gmbh. The BeanDevice AX-3D wireless accelerometer sensors were used to measure the dynamic responses of the vehicle. The sensor was installed on the front and rear axles of the vehicle as shown in Figure 9(b). Laser sensors were installed to record the time instants when the vehicle arrives at and exits from the main beam. These time instants were used to calculate the moving speed of the vehicle. The sample frequency for the measurement was $500 \mathrm{~Hz}$.

Plastic strip with three kinds of thickness were attached to the surface of the beam to simulate bumps on the road as shown in Figure 10. The thickness and locations of the strips installed were summarized in Table 3. The ' $1 / 8$ ' in Table 3 denotes the location of the strip at $1 / 8$ length of the first main span from its left support.

Figure 11 shows the measured responses of the vehicle when it is passing over the main beam with a speed $0.35 \mathrm{~m} / \mathrm{s}$. The proposed method is used to identify the interaction force from the vehicle responses with $\boldsymbol{Q}^{\boldsymbol{p}}=1 \times 10^{20}$. The identified dynamic interaction forces are compared with that are calculated directly using the measured acceleration responses and integration technique and the results are shown in Figure 12(a). The two sets of forces match each other quite well. The spectra of the identified force in Figure 12(b) has two dominant peaks at 29.4 and $36.7 \mathrm{~Hz}$ corresponding to the two modal frequencies of the vehicle model. The apparent profile is defined as the profile of displacement at the contact point between the tire and the bridge surface [34] and it is calculated using 
the interaction forces and the identified displacement at the top of the two axles. A band pass filter with cut-off frequencies at 1.0 to $100.0 \mathrm{~Hz}$ is used to process the apparent profile to estimate the beam surface unevenness. The identified unevenness from two vehicle axles are shown in Figure 13. The locations of the installed plastic strips were all identified and the identified values are presented in Table 3. The identified results from the front and rear axles are very consistent and the values from the front axle are generally larger than those from the rear axle.

\section{Studies on structural damage detection}

The same vehicle-bridge interaction system for numerical studies in Section 5 is considered, and local damages are introduced into the finite elements of the bridge model. Two damage cases are studied with (a) single damage scenario - $20 \%$ damage is introduced in Element 2, i.e. $\alpha_{s}^{2}=0.20$; and (b) multiple damage case - Elements 4 and 8 are assumed to have damage with $\alpha_{s}^{4}=0.15, \alpha_{s}^{8}=0.20$. The algorithm described in Section 4 is adopted for the damage identification with the tolerance in Eq. (26) equal to $5 \times 10^{-4}$ in the numerical example.

\subsubsection{Damage detection with a smooth bridge surface}

Five percent measurement noise is added into the calculated vehicle responses. The cases with three different vehicle moving speeds, i.e. 10, 20 and 30m/s, are studied. As shown in Figure 14, the identified results show that the damage location are identified correctly for all vehicle speeds. The identified damage extents are very close to the true values. However, a lower vehicle speed is noted to give better identification results when the bridge surface is smooth.

To further study the effect of measurement noise on the identification, another example is studied with $10 \%$ measurement noise and $20 \mathrm{~m} / \mathrm{s}$ moving speed. The identified results are compared with those for 5\% measurement noise, as shown in Figure 15. The damage location and extent are noted accurately identified. It may therefore concluded that when the bridge surface is smooth, the proposed method can be used for drive-by bridge damage detection with a high vehicle speed using noisy vehicle axle responses.

\subsubsection{Damage detection when the bridge surface roughness is known}

The proposed method is used for the damage detection with the identified forces. Section 5.1.2 has shown the measurement noise effect is significant to the identified results, and therefore a higher 
sampling rate of $5000 \mathrm{~Hz}$ is used. The Classes A and B bridge surface roughness are adopted. 5\% measurement noise and a moving speed of $20 \mathrm{~m} / \mathrm{s}$ are considered. The identified results are shown in Figure 16. When the bridge surface roughness is known, the interaction forces obtained from the vehicle axle responses with the proposed method can yield damage location and extent with acceptable accuracy. Some elements are, however, falsely identified with small stiffness variation which is due to the effect of measurement noise. The proposed method can provide reliable information on potential damage conditions of the structure when the bridge surface roughness is known.

\subsubsection{Damage detection using the identified bridge surface roughness}

When both the interaction forces and vehicle displacements are identified from the vehicle axle responses, the surface roughness can be calculated from the known vehicle-bridge system. The moving speed $30 \mathrm{~m} / \mathrm{s}$ and the sampling frequency $5000 \mathrm{~Hz}$ are adopted. The damage detection results considering different measurement noise levels are shown in Figure 17. When the measurement noise is $2 \%$, the identified damage location and extent are acceptable. For a higher noise level of $5 \%$, a few false detection are noted with the designated damaged elements identified with much higher possibility of damage. When the noise level reaches $10 \%$, the identified damage information are not reliable for the single damage case. The measurement noise is noted to have large effect on the surface roughness estimation leading to an inaccurate damage identification. The advanced signal preprocessing is needed to reduce the measurement noise when the proposed method is applied in the practice. The results show that the bridge damage could be identified with the predicted road surface roughness using the intact bridge model. In practice, the bridge condition is unknown. The bridge road surface roughness and damage could be identified simultaneously using the iterative procedure with the proposed two-step approach.

\section{Conclusions}

Vehicle-bridge interaction force identification and bridge damage detection using vehicle responses are two techniques with great potential of application. Both require only instrumentation on the moving vehicle. A state space model of the vehicle based on Newmark-beta formulation is derived in this paper. Dual Kalman filter is used to identify the interaction forces between the vehicle and bridge with only the vehicle axle responses. The bridge surface roughness can be calculated using the interaction forces and bridge parameters. A method is proposed to estimate the forces and road surface roughness with measurement noise in the responses simultaneously through the iterative procedure. 
The interaction forces obtained can then be used for bridge damage detection based on the interaction force sensitivity analysis. Numerical examples show that the damage index of the bridge deck can be identified from the interaction forces effectively when the measurement noise is less than $5 \%$. Experimental investigation is also conducted to verify the feasibility of the proposed method for interaction force and bridge surface roughness estimation. Further experimental study is required to reduce the effect of measurement noise on the damage identification for practical application.

\section{Acknowledgements}

This research is supported by research funding of the Australian Research Council Discover Project (DP160103197). The financial aid is gratefully acknowledged.

\section{Appendix I}

$$
\begin{aligned}
& \mathbf{M}_{v 1}=\left[\begin{array}{cc}
m_{v} & 0 \\
0 & I_{v}
\end{array}\right], \mathbf{M}_{v 2}=\left[\begin{array}{cc}
m_{1} & 0 \\
0 & m_{2}
\end{array}\right], \mathbf{C}_{v 11}=\left[\begin{array}{cc}
C_{s 1}+C_{s 2} & \left(-C_{s 1} a_{1}+C_{s 2} a_{2}\right) S \\
\left(-C_{s 1} a_{1}+C_{s 2} a_{2}\right) S & \left(C_{s 1} a_{1}^{2}+C_{s 2} a_{2}^{2}\right) S^{2}
\end{array}\right], \\
& \mathbf{C}_{v 12}=\left[\begin{array}{cc}
-C_{s 1} & -C_{s 2} \\
C_{s 1} a_{1} S & -C_{s 2} a_{2} S
\end{array}\right], \mathbf{C}_{v 21}=\left[\begin{array}{cc}
-C_{s 1} & C_{s 1} a_{1} S \\
-C_{s 2} & -C_{s 2} a_{2} S
\end{array}\right], \mathbf{C}_{v 22}=\left[\begin{array}{cc}
C_{s 1} & 0 \\
0 & C_{s 2}
\end{array}\right], \\
& \mathbf{K}_{v 11}=\left[\begin{array}{cc}
K_{s 1}+K_{s 2} & \left(-K_{s 1} a_{1}+K_{s 2} a_{2}\right) S \\
\left(-K_{s 1} a_{1}+K_{s 2} a_{2}\right) S & \left(K_{s 1} a_{1}^{2}+K_{s 2} a_{2}^{2}\right) S^{2}
\end{array}\right], \mathbf{K}_{v 12}=\left[\begin{array}{cc}
-K_{s 1} & -K_{s 2} \\
K_{s 1} a_{1} S & -K_{s 2} a_{2} S
\end{array}\right], \\
& \mathbf{K}_{v 21}=\left[\begin{array}{ll}
-K_{s 1} & K_{s 1} a_{1} S \\
-K_{s 2} & -K_{s 2} a_{2} S
\end{array}\right], \mathbf{K}_{v 22}=\left[\begin{array}{cc}
K_{s 1} & 0 \\
0 & K_{s 2}
\end{array}\right] . \\
& P_{i}(t)=K_{t i}\left(y_{i}-z_{i}\right)+C_{t i}\left(\dot{y}_{i}-\dot{z}_{i}\right)+\left\{m_{i}+\left(1-a_{i}\right) m_{v}\right\} g \\
& z_{i}=w\left(\hat{x}_{i}(t), t\right)+r\left(\hat{x}_{i}(t)\right), \dot{z}_{i}=\dot{w}\left(\hat{x}_{i}(t), t\right)+w^{\prime}\left(\hat{x}_{i}(t), t\right) \dot{\hat{x}}_{i}(t)+r^{\prime}\left(\hat{x}_{i}(t)\right) \dot{\hat{x}}_{i}(t), \\
& \dot{w}\left(\hat{x}_{i}(t), t\right)=\mathbf{H}_{c}^{T}\left(\hat{x}_{i}(t)\right) \dot{\mathbf{d}}_{b}(t), \quad w^{\prime}\left(\hat{x}_{i}(t), t\right)=\left.\frac{\partial \mathbf{H}_{c}^{T}(x)}{\partial x} \mathbf{d}_{b}(t)\right|_{x=\hat{x}_{i}(t),} \\
& r^{\prime}\left(\hat{x}_{i}(t)\right)=\left.\frac{d r(x)}{d x}\right|_{x=\hat{x}_{i}(t),} \quad \dot{\hat{x}}_{i}(t)=\frac{d \hat{x}_{i}(t)}{d t} . \quad(i=1,2) \\
& \left\{\begin{array}{l}
M_{s 1}=\left(m_{1}+a_{2} m_{v}\right) \mathrm{g} \\
M_{s 2}=\left(m_{2}+a_{1} m_{v}\right) \mathrm{g}
\end{array}\right.
\end{aligned}
$$

where $r(x)$ is the road surface roughness at the location of the tires; $\hat{x}_{1}(t)$ and $\hat{x}_{2}(t)$ are the position of the front and rear axles respectively at time $t . m_{v}, I_{v}, m_{1}, m_{2}, C_{s 1}, C_{s 2}, C_{t 1}, C_{t 2}, K_{s 1}, K_{s 2}, K_{t 1}$, $K_{t 2}$ are the vehicle parameters as shown in Figure 1. $a_{1} S, a_{2} S$ are the distance between the center of gravity of the vehicle body with the front and rear axles respectively and $S$ is the axle spacing. $g$ is 
the acceleration of gravity. $w\left(\hat{x}_{i}(t), t\right), \dot{w}\left(\hat{x}_{i}(t), t\right)(i=1,2)$ are the vertical dynamic deflection of the beam and its time derivative under the $i$ th load at time $t . z_{i}(i=1,2)$ is the displacements under the $i$ th tire.

$\mathbf{M}_{g}=\left[\begin{array}{ccc}\mathbf{M}_{b} & \mathbf{0} & \mathbf{H}_{c} \mathbf{M}_{v 2} \\ \mathbf{0} & \mathbf{M}_{v 1} & \mathbf{0} \\ \mathbf{0} & \mathbf{0} & \mathbf{M}_{v 2}\end{array}\right], \mathbf{C}_{g}=\left[\begin{array}{ccc}\mathbf{C}_{b} & \mathbf{H}_{c} \mathbf{C}_{v 21} & \mathbf{H}_{c} \mathbf{C}_{v 22} \\ \mathbf{0} & \mathbf{C}_{v 11} & \mathbf{C}_{v 12} \\ \mathbf{H}_{c}{ }^{T} \mathbf{C}_{t} & \mathbf{C}_{v 21} & \mathbf{C}_{v 22}+\mathbf{C}_{t}\end{array}\right]$,

$\mathbf{K}_{g}=\left[\begin{array}{ccc}\mathbf{K}_{b} & \mathbf{H}_{c} \mathbf{K}_{v 21} & \mathbf{H}_{c} \mathbf{K}_{v 22} \\ \mathbf{0} & \mathbf{K}_{v 11} & \mathbf{K}_{v 12} \\ \mathbf{H}_{c}{ }^{T} \mathbf{K}_{t}+\mathbf{H}_{c}^{\prime T} \mathbf{C}_{t} & \mathbf{K}_{v 21} & \mathbf{K}_{v 22}+\mathbf{K}_{t}\end{array}\right]$

$\mathbf{P}_{\boldsymbol{g}}=\left[\begin{array}{lll}\mathbf{H}_{c} \mathbf{M}_{s} & \mathbf{0} & \boldsymbol{p}\end{array}\right]^{T}$,

$\boldsymbol{p}(\boldsymbol{t})=\left\{C_{t 1} r^{\prime}\left(\hat{x}_{1}(t)\right) \dot{\hat{x}}_{1}(t)+K_{t 1} r\left(\hat{x}_{1}(t)\right) \quad C_{t 2} r^{\prime}\left(\hat{x}_{2}(t)\right) \dot{\hat{x}}_{2}(t)+K_{t 2} r\left(\hat{x}_{2}(t)\right)\right\}^{T}$

where $\mathbf{C}_{t}=\operatorname{diag}\left(C_{t 1}, C_{t 2}\right)$ and $\mathbf{K}_{t}=\operatorname{diag}\left(K_{t 1}, K_{t 2}\right)$.

\section{References}

[1] Dahlberg T (1984) Vehicle-bridge interaction. Vehicle System Dynamics 13(4): 187-206.

[2] Yang YB and Lin CW (2005) Vehicle-bridge interaction dynamics and potential applications. Journal of Sound and Vibration 284(1-2): 205-226.

[3] Li HY, Wekezer J and Malachowski J (2006) Finite element analysis of vehicle-bridge interaction. Finite Elements in Analysis and Design 42(11): 950-959.

[4] Lu F, Lin JH, Kennedy D and Williams FW (2009) An algorithm to study non-stationary random vibrations of vehicle-bridge systems. Computer and Structures 87: 177-185.

[5] Kim JH, Lynch JP, Lee JJ and Lee CC (2011) Truck-based mobile wireless sensor networks for the experimental observation of vehicle-bridge interaction. Smart Materials and Structures 20(6): 065009

[6] Lombaert G and Conte JP (2012) Random vibration analysis of dynamic vehicle-bridge interaction due to road unevenness. Journal of Engineering Mechanics ASCE 138(7): 816-825.

[7] Chen, S.Y., Xia, H. and Zhang, J.W. (2007) Review on bridge structural damage identification techniques based on bridge-vehicle coupled vibration analysis. China Safety Science Journal 17: 148-155.

[8] Zhu, XQ and Law, SS (2015) Structural health monitoring based on vehicle-bridge interaction: accomplishment and challenges. Advances in Structural Engineering 18(12):1999-2015.

[9] Yang YB, Lin CW and Yau JD (2004) Extracting bridge frequencies from the dynamic response of a passing vehicle. Journal of Sound and Vibration 272(3): 471-93.

[10] $\mathrm{Bu}$ JQ, Law SS \& Zhu XQ (2006) Innovative bridge condition assessment from dynamic response of a passing vehicle. Journal of Engineering Mechanics 132(12):1372-9. 
[11] Zhang Y, Lie ST and Xiang ZH (2013) Damage detection method based on operating deflection shape curvature extracted from dynamic response of a passing vehicle. Mechanical Systems and Signal Processing 35(1): 238-54.

[12] Kong X, Cai C and Kong B (2014) Damage detection based on transmissibility of a vehicle and bridge coupled system. Journal of Engineering Mechanics 141(1): 04014102.

[13] Li ZH and Au FTK (2014) Damage detection of a continuous bridge from response of a moving vehicle. Shock and Vibration 2014: 146802.

[14] Yang YB and Yang JP (2018) State-of-the-art review on modal identification and damage detection of bridges by moving test vehicles. International Journal of Structural Stability and Dynamics 18(2): 1850025

[15] Zhu XQ, Law SS, Huang L, and Zhu SY (2018) Damage identification of supporting structures with a moving sensory system. Journal of Sound and Vibration 415:111-127.

[16] Zhang B, Qian Y, Wu YT and Yang YB (2018) An effective means for damage detection of bridges using the contact-point response of a moving test vehicle. Journal of Sound and Vibration 419: $158-172$.

[17] Pan CD, Yu L, Liu HL, Chen ZP, and Luo WF (2018) Moving force identification based on redundant concatenated dictionary and weighted 11-norm regularization. Mechanical Systems and Signal Processing 98: 32-49.

[18] Zhong J, Liu H and Yu L (2019) Sparse regularization for traffic load monitoring using bridge response measurements. Measurement 131: 173-182.

[19] Pan CD, Liu HL and Yu L (2019) A sparse self-estimated sensor-network for reconstructing moving vehicle forces. Smart Materials and Structures 28: 085009.

[20] O'Brien E, McGetrick P and Gonzalez A (2014) A drive-by inspection system via vehicle moving force identification. Smart Structures and Systems 13: 821-848.

[21] Yang JN, Lin S, Huang HW, Zhou L(2006) An adaptive extended Kalman filter for structural damage identification, Journal of Structural Control and Health Monitoring 13:849-867.

[22] Petersen CD, Fraanje R, Cazzolato BS, Zander AC, and Hansen CH (2008) A Kalman filter approach to virtual sensing for active noise control. Mechanical Systems and Signal Processing 22: 490-508.

[23] Liu LJ, Zhu JJ, Su Y and Lei Y. (2016) Improved Kalman filter with unknown inputs based on data fusion of partial acceleration and displacement measurements. Smart Structures and Systems 17(6): 903-915.

[24] Lei Y, Jiang Y and Xu Z. (2012) Structural damage detection with limited input and output measurement signals. Mechanical Systems and Signal Processing 28: 229-243.

[25] Calabrese A, Strano S and Terzo M. (2018) Adaptive constrained unscented Kalman filtering for real-time nonlinear structural system identification. Structural Control and Health Monitoring 25: e2084.

[26] Lei Y, Xia DD, Erazo K and Nagarajaiah S A novel unscented Kalman filter for recursive stateinput-system identification of nonlinear systems. Mechanical Systems and Signal Processing 127: 120-135.

[27] Erazo K, Sen D, Nagarajaiah S, and Sun LM (2019) Vibration-based structural health monitoring under changing environmental conditions using Kalman filtering. Mechanical Systems and Signal Processing 117: 1-15. 
[28] Aucejo M, De Smet O, Deü JF (2019) Practical issues on the applicability of Kalman filtering for reconstructing mechanical sources in structural dynamics. Journal of Sound and Vibration 442:48-70.

[29] Azam SE, Chatzi E and Papadimitriou C (2015) A dual Kalman filter approach for state estimation via output-only acceleration measurements. Mechanical Systems and Signal Processing 60-61: 866-886.

[30] Liu K, Law SS, Zhu XQ, and Xia Y (2014) Explicit form of an implicit method for inverse force identification. Journal of Sound and Vibration 333(3): 730-44.

[31] Henchi k, Fafard M, Talbot M, and Dhatt G. (1998) An efficient algorithm for dynamic analysis of bridges under moving vehicles using a coupled modal and physical components approach. Journal of Sound and Vibration 242: 663-83.

[32] ISO-8608 1995, Mechanical vibration-Road surface profiles-Reporting of measured data, International Organization for Standardization (ISO) Geneva.

[33] Hansen PC. (1992) Analysis of discrete ill-posed problems by means of the L-curve. SIAM review 34: 561-580.

[34] ElHattab A, Uddin N and O'Brien E. (2017) Drive-by bridge damage detection using nonspecialized instrumented vehicle. Bridge Structures 12: 73-84. 
Table 1 RPE of the identified results considering different measurement noise $(\%)$

\begin{tabular}{|c|c|c|c|c|}
\hline Noise level (\%) & \multicolumn{2}{|c|}{ Forces } & \multicolumn{2}{c|}{ Roughness } \\
\hline & Front & Rear & Front & Rear \\
\hline 0 & 0.00 & 0.00 & 2.61 & 2.61 \\
\hline 2 & 2.06 & 3.25 & 6.86 & 10.41 \\
\hline 5 & 5.88 & 6.79 & 18.30 & 17.98 \\
\hline 10 & 11.75 & 13.59 & 36.3 & 35.72 \\
\hline
\end{tabular}

Table 2 RPE of the identified results considering different vehicle speed (\%)

\begin{tabular}{|c|c|c|c|c|c|c|c|c|}
\hline & \multicolumn{4}{|c|}{ Sampling frequency=1000 Hz } & \multicolumn{3}{c|}{ Sampling frequency $=5000 \mathrm{~Hz}$} \\
\hline $\begin{array}{c}\text { Moving } \\
\text { speed (m/s) }\end{array}$ & \multicolumn{2}{|c|}{ Forces } & \multicolumn{2}{c|}{ Roughness } & \multicolumn{2}{c|}{ Forces } & \multicolumn{2}{c|}{ Roughness } \\
\hline & Front & Rear & Front & Rear & Front & Rear & Front & Rear \\
\hline 10 & 4.78 & 2.79 & 52.97 & 52.36 & 3.07 & 1.72 & 34.81 & 40.84 \\
\hline 20 & 5.88 & 6.79 & 18.30 & 17.98 & 4.04 & 4.74 & 13.74 & 27.71 \\
\hline 30 & 5.12 & 8.98 & 5.23 & 8.68 & 4.14 & 5.96 & 4.91 & 2.91 \\
\hline
\end{tabular}

Table 3. The experimental setup of the beam surface unevenness and the identification results

\begin{tabular}{|c|c|c|c|c|c|c|c|c|}
\hline \multicolumn{8}{|c|}{ Experimental setup } \\
\hline Label & 1 & 2 & 3 & 4 & 5 & 6 & 7 & 8 \\
\hline Locations & $1 / 8$ & $1 / 4$ & $5 / 16$ & $3 / 8$ & $1 / 2$ & $5 / 8$ & $3 / 4$ & $7 / 8$ \\
\hline Layer & 1 & 1 & 1 & 1 & Thinner & 2 & 2 & 1 \\
\hline \multicolumn{7}{|c|}{ Identification results } \\
\hline $\begin{array}{c}\text { Identified from } \\
\text { front axle (mm) }\end{array}$ & 2.00 & 1.23 & 2.36 & 1.69 & 1.04 & 2.93 & 3.13 & 1.50 \\
\hline $\begin{array}{c}\text { Identified from } \\
\text { rear axle (mm) }\end{array}$ & 1.63 & 1.37 & 1.87 & 1.53 & 1.02 & 2.50 & 2.66 & 1.44 \\
\hline
\end{tabular}




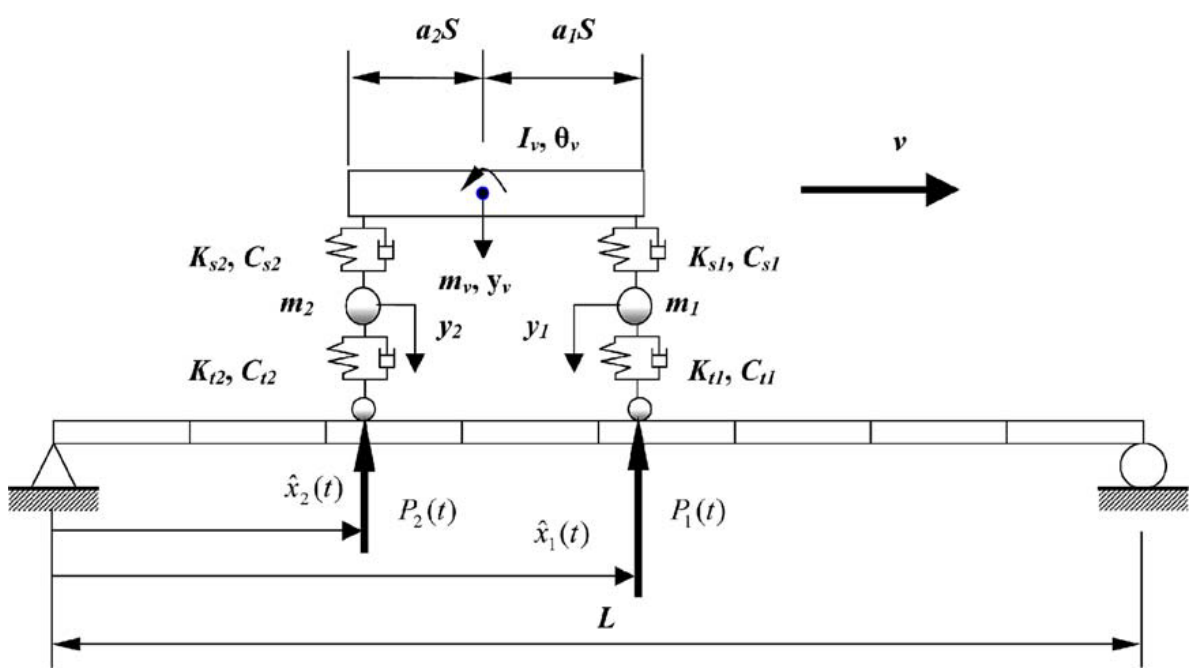

Figure 1 Vehicle-bridge interaction model

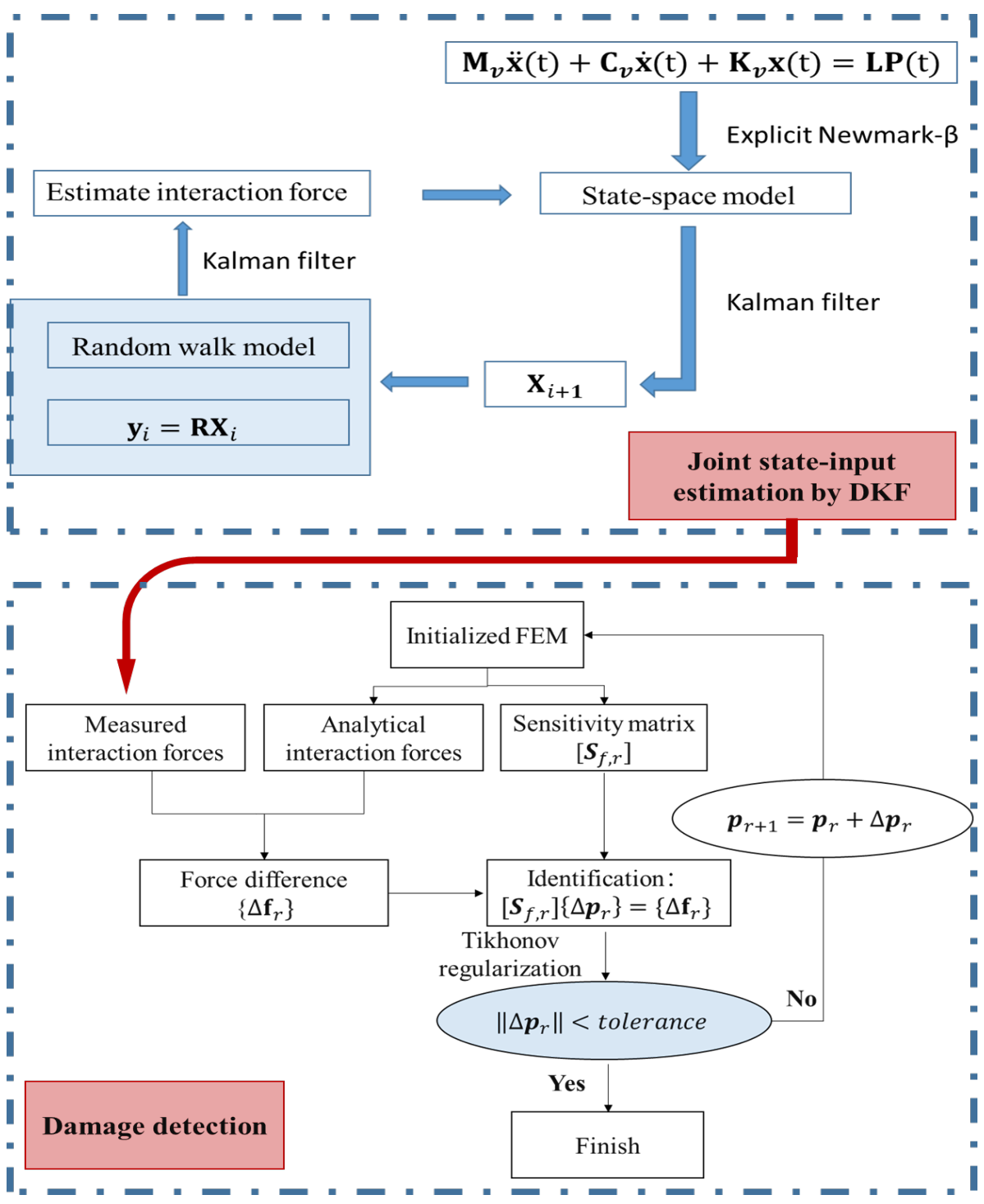

Figure 2 Flow chart of proposed method 

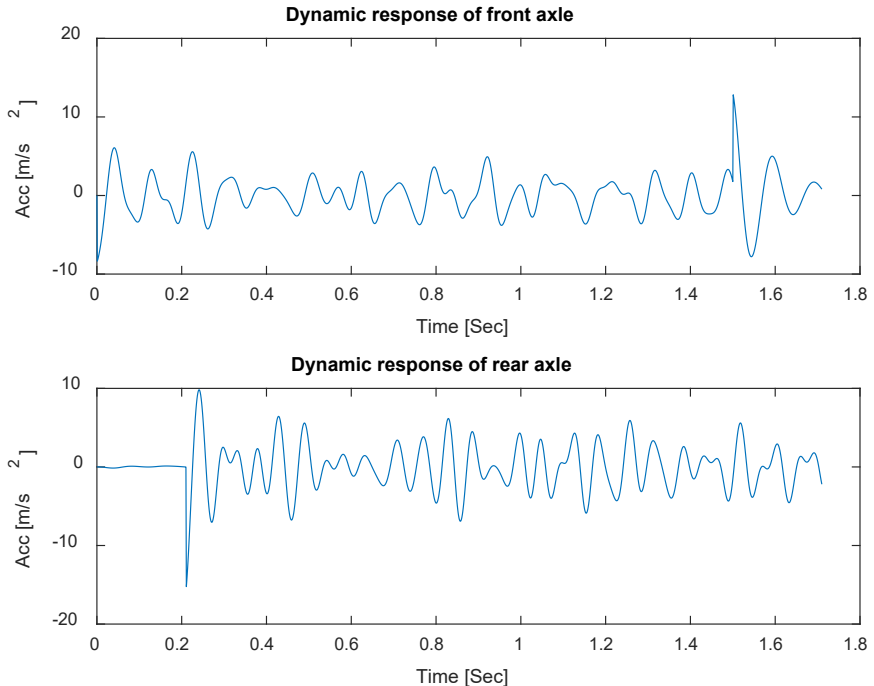

Figure 3 Axles responses

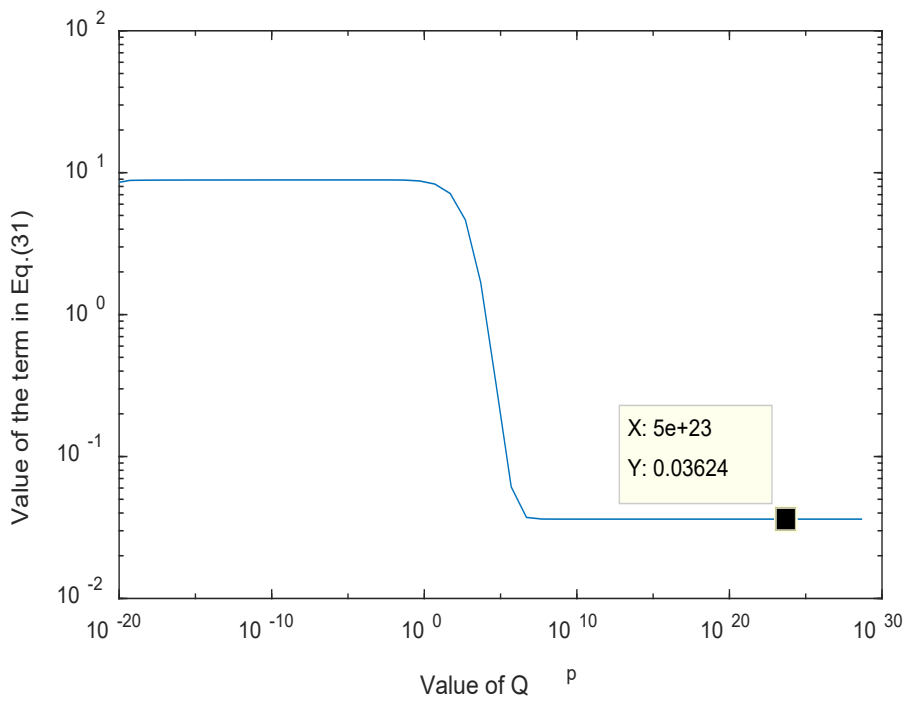

Figure 4 Innovation norm and location of the minimum value 

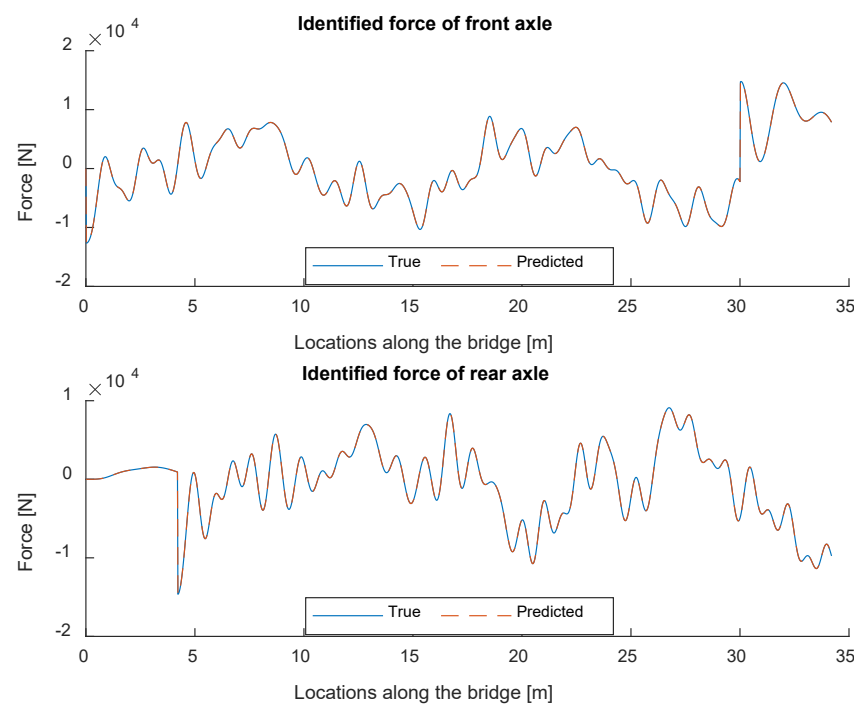

(a) Identified interaction forces

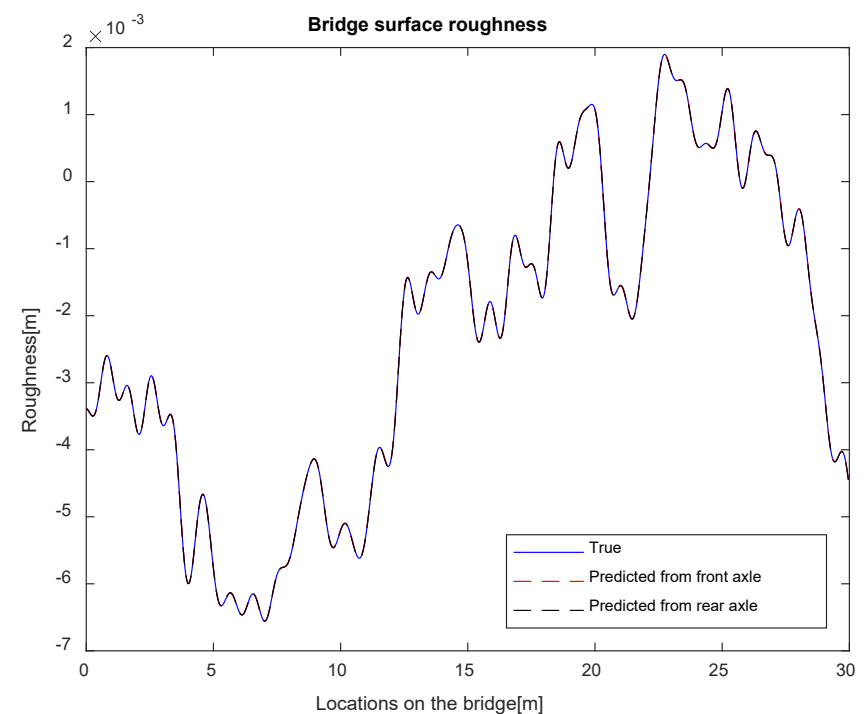

(b) Identified bridge surface roughness

Figure 5 Identified results with Class B roughness and $v=20 \mathrm{~m} / \mathrm{s}$ 

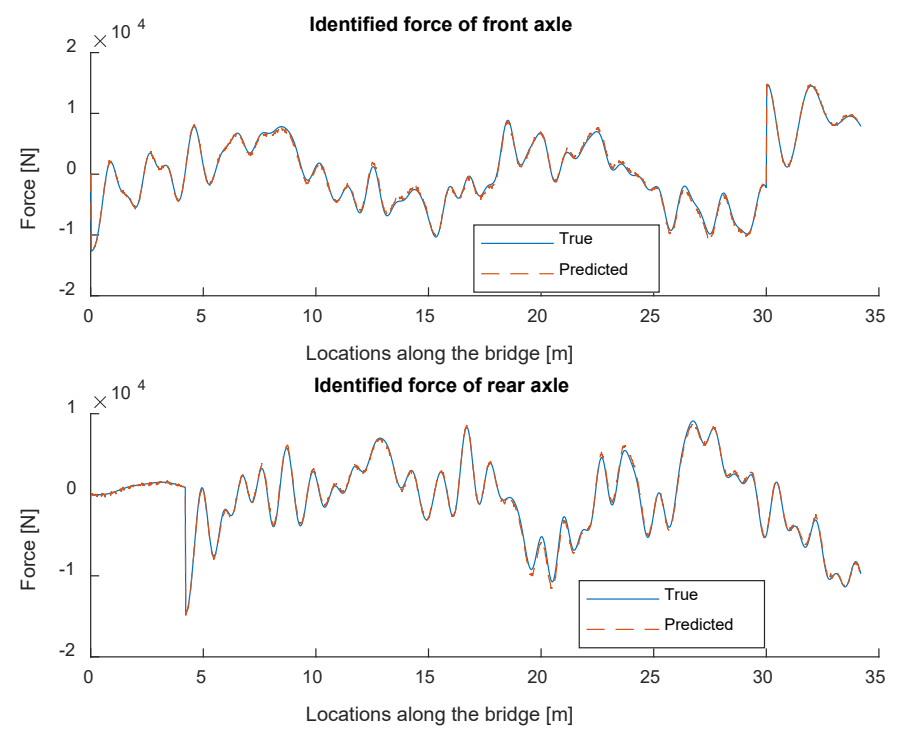

(a) Identified interacting axle forces when 5\% measurement noise is added

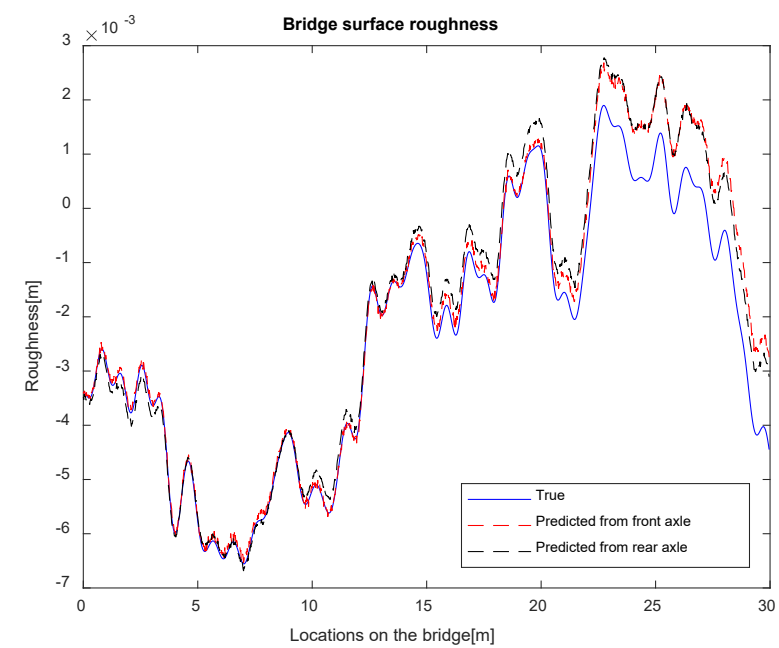

(b) Identified bridge surface roughness

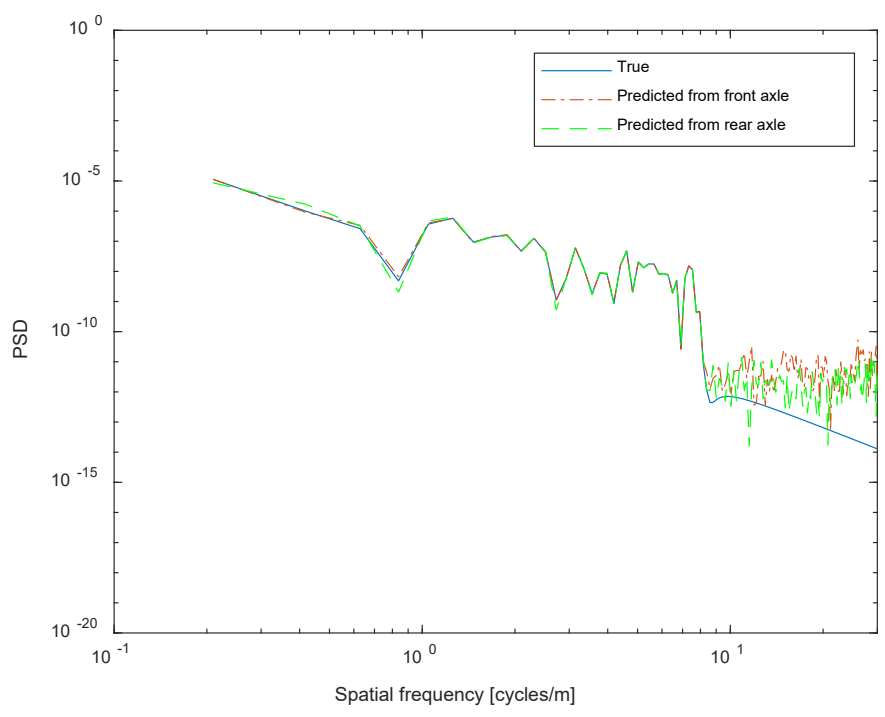

(c) PSD of surface roughness

Figure 6 Identified forces and surface roughness with 5\% measurement noise 

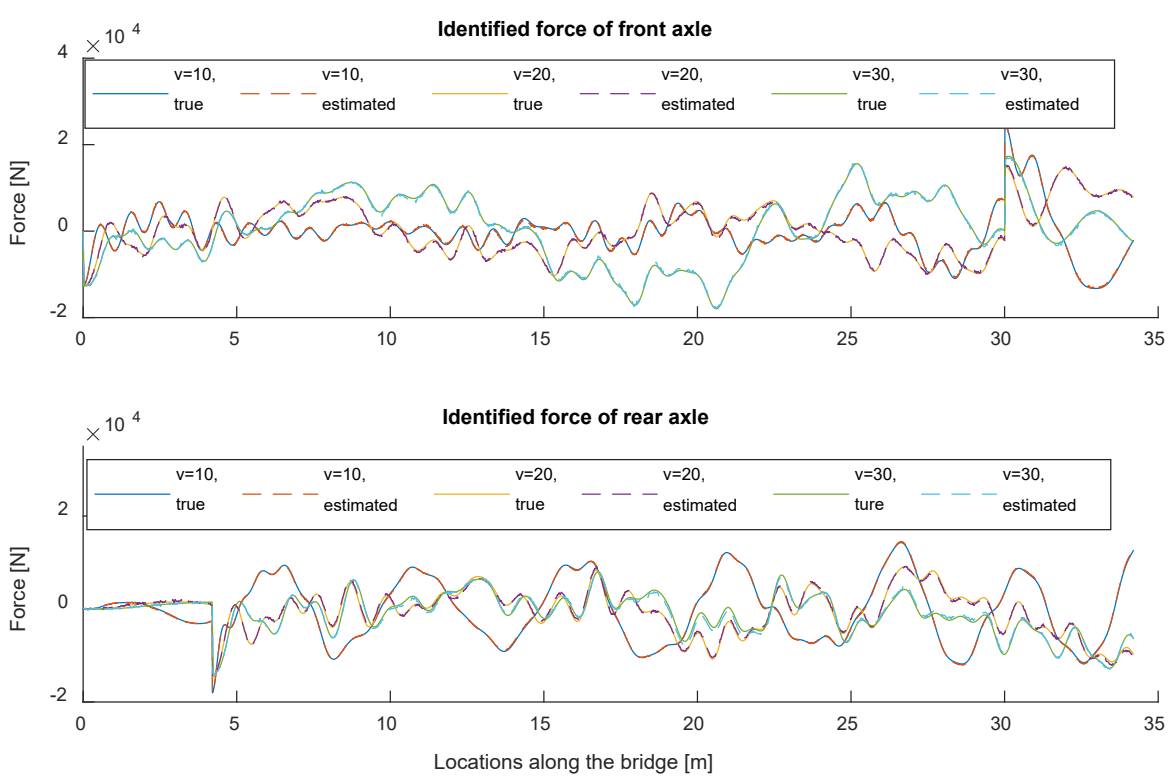

(a) Identified interaction forces
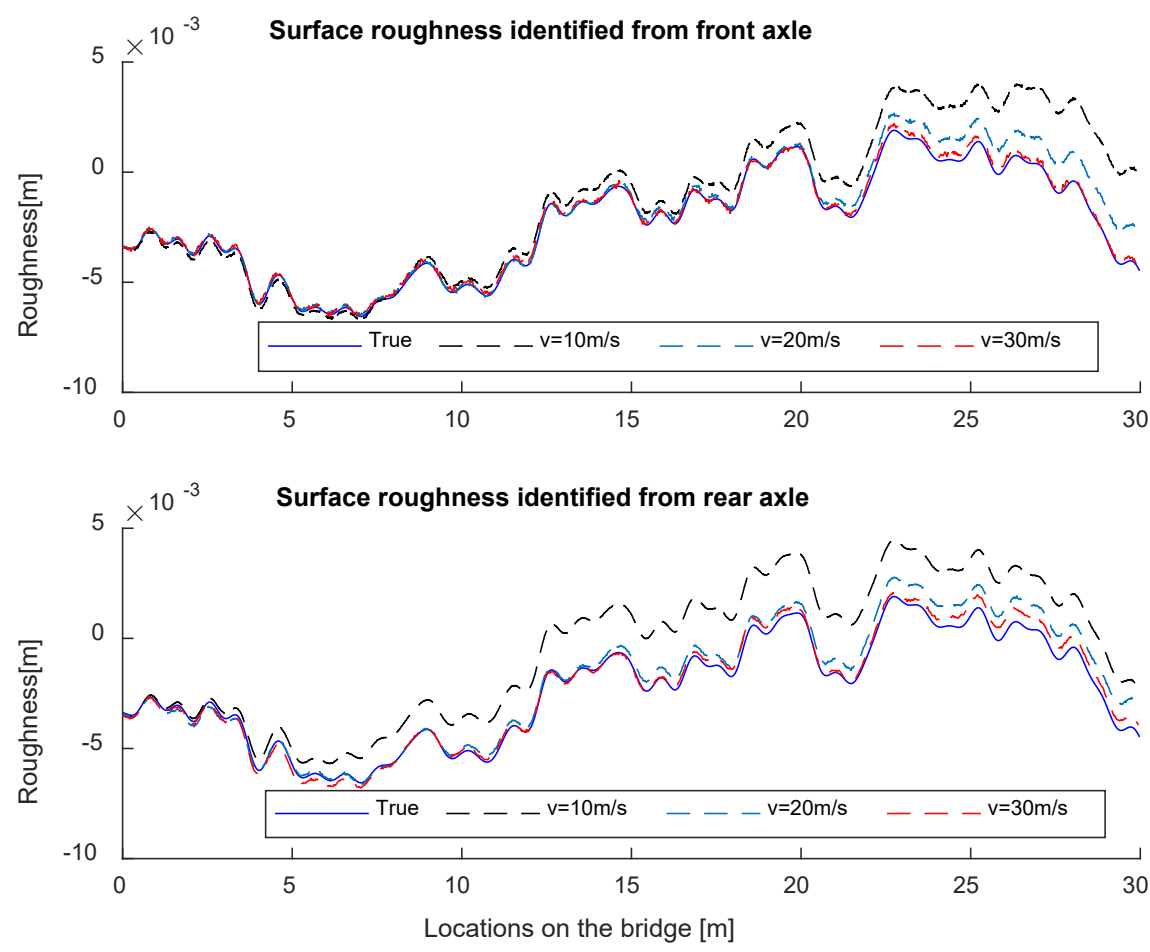

(b) Identified bridge surface roughness

Figure 7 Identified results considering different moving speed with $\mathrm{Fs}=1000 \mathrm{~Hz}$ 

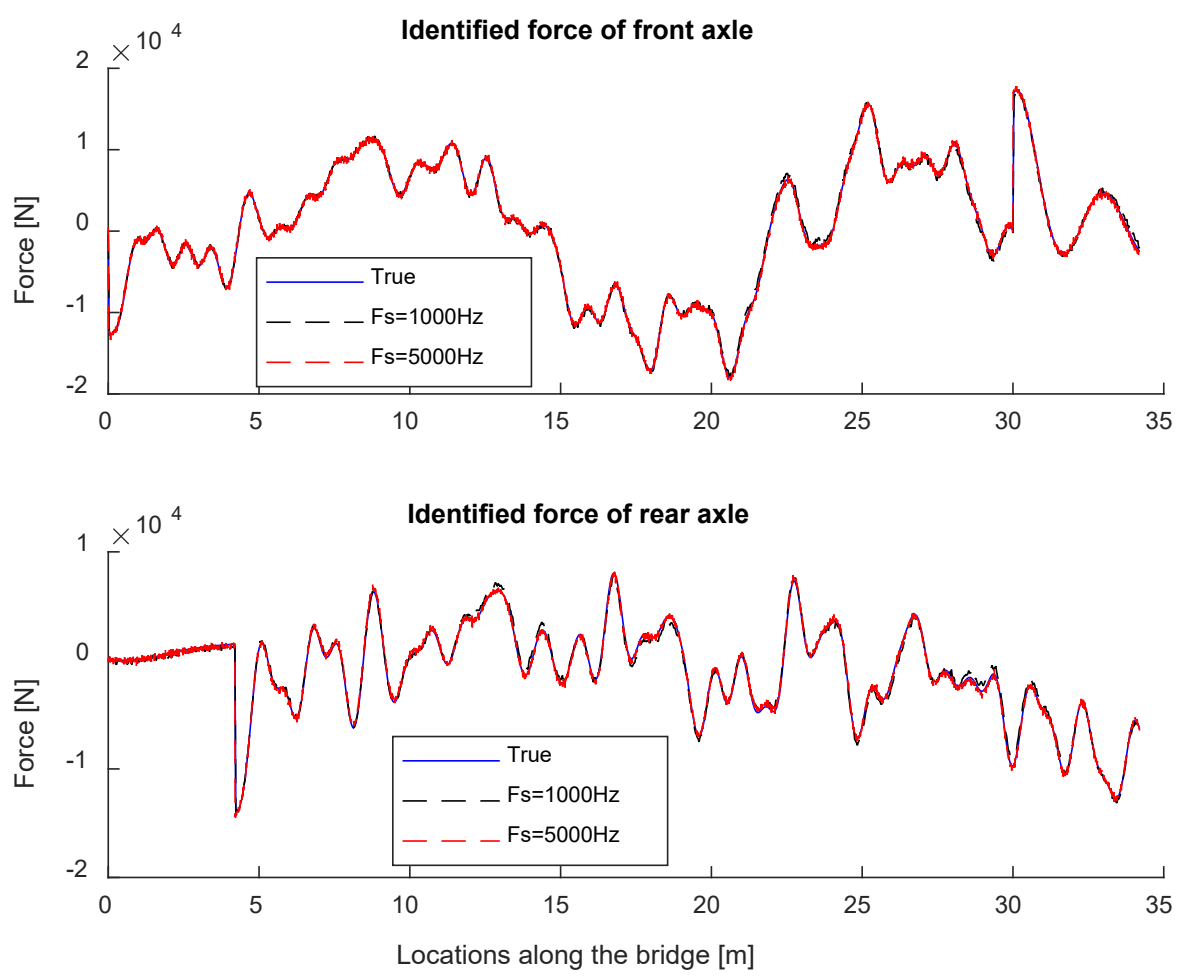

(a) Identified interaction forces
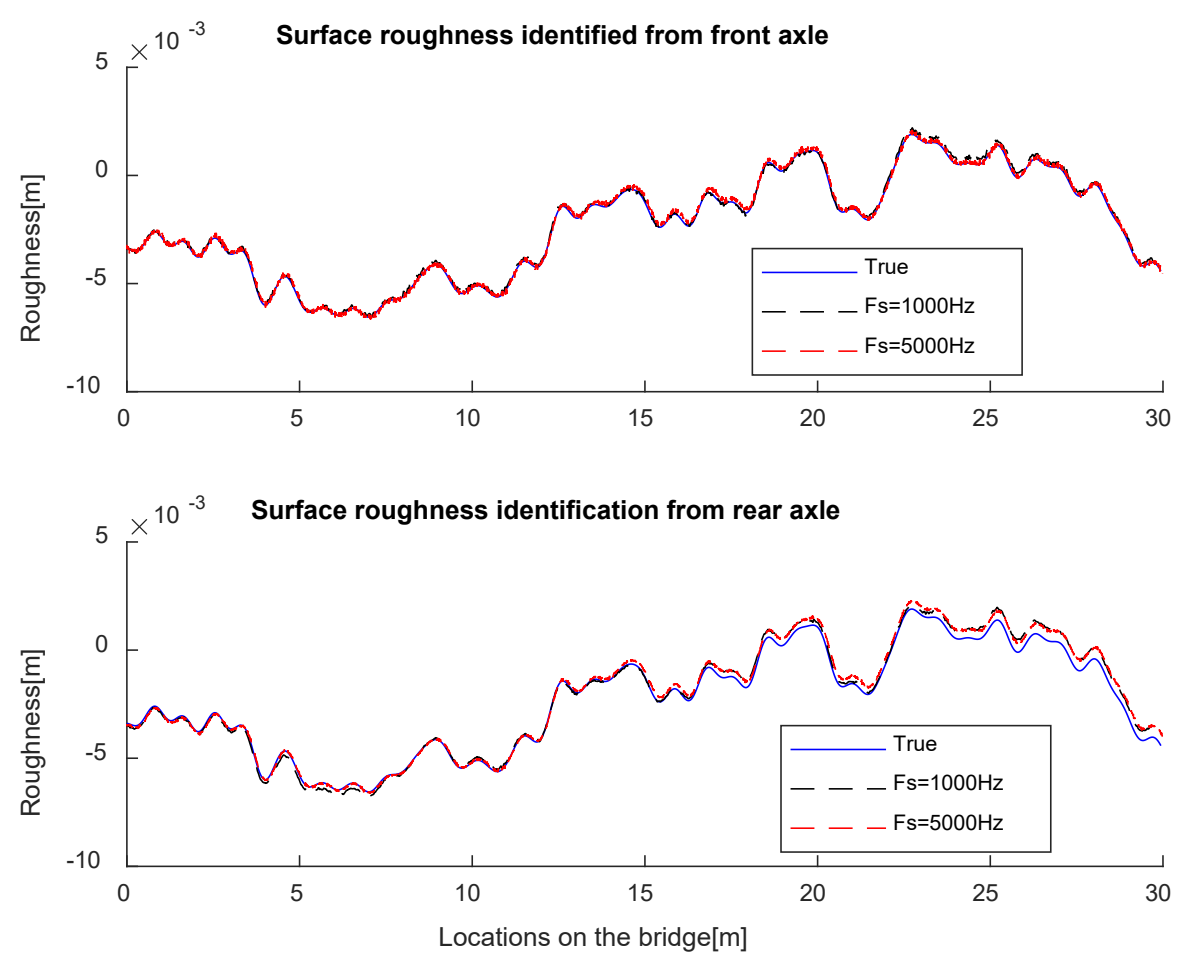

(b) Identified bridge surface roughness

Figure 8 Identified results considering different sampling frequency with $\mathrm{v}=30 \mathrm{~m} / \mathrm{s}$ 


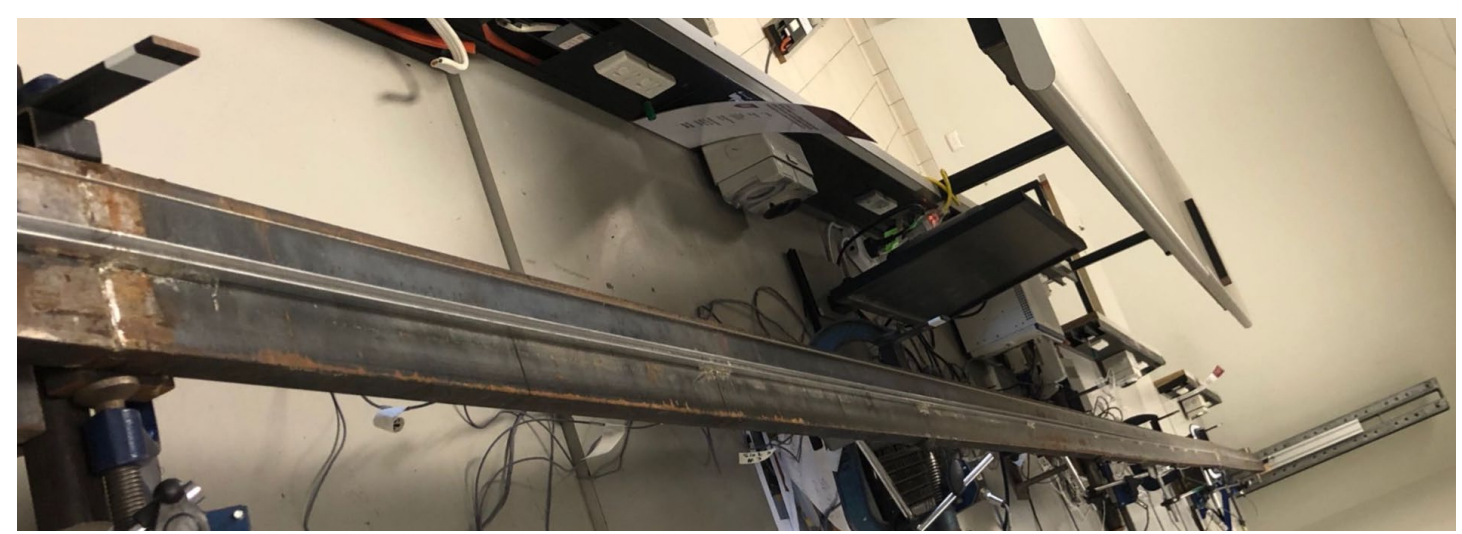

(a) The bridge model

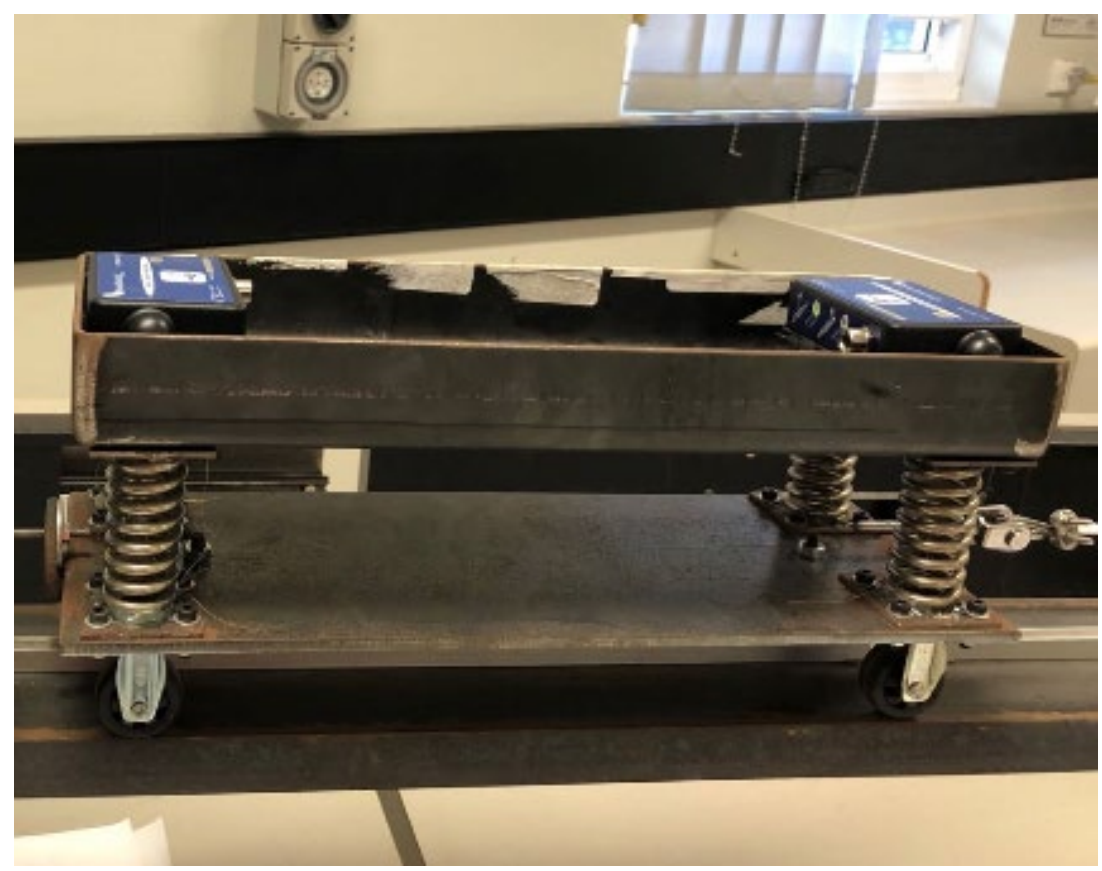

(b) The vehicle model

Figure 9 Vehicle-bridge interaction model in the lab

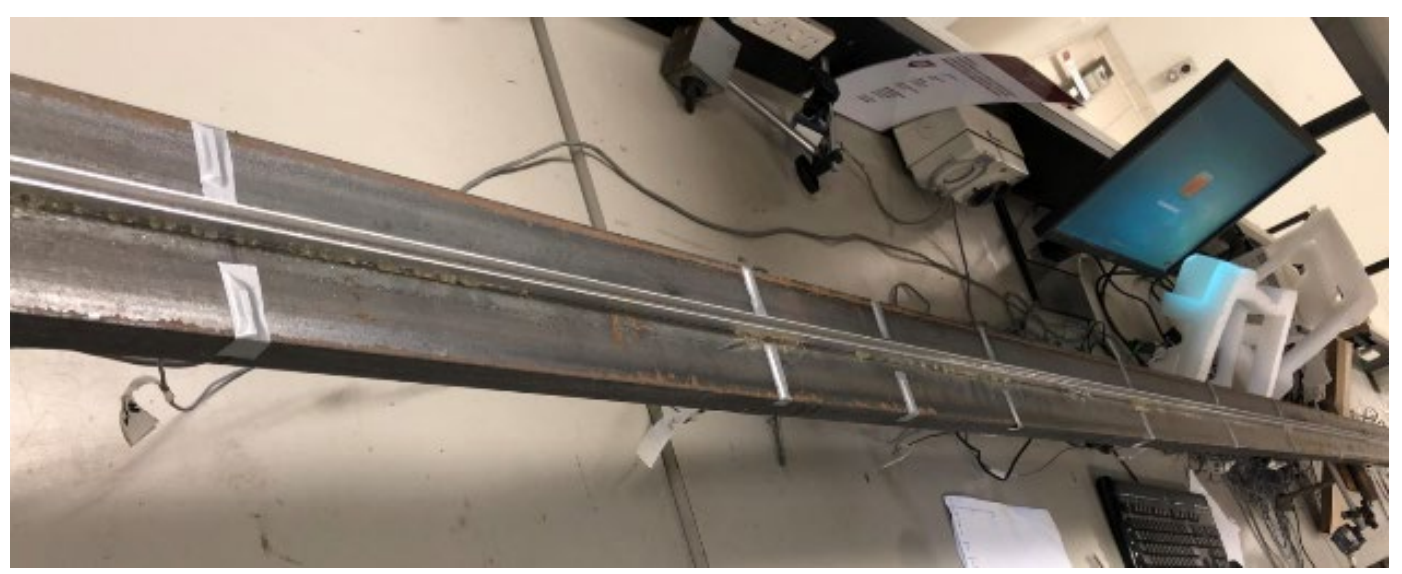

Figure 10 The installation of plastic strip to simulate bumps on the bridge 

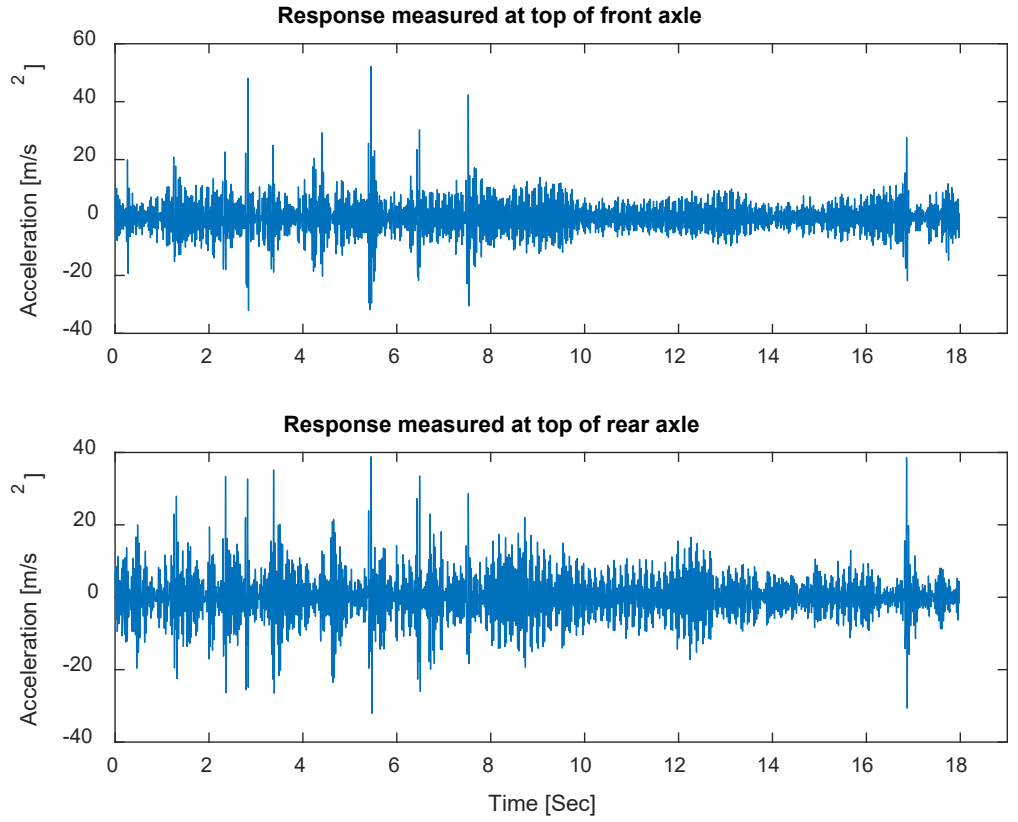

Figure 11 Measured responses from wireless sensors on the vehicle
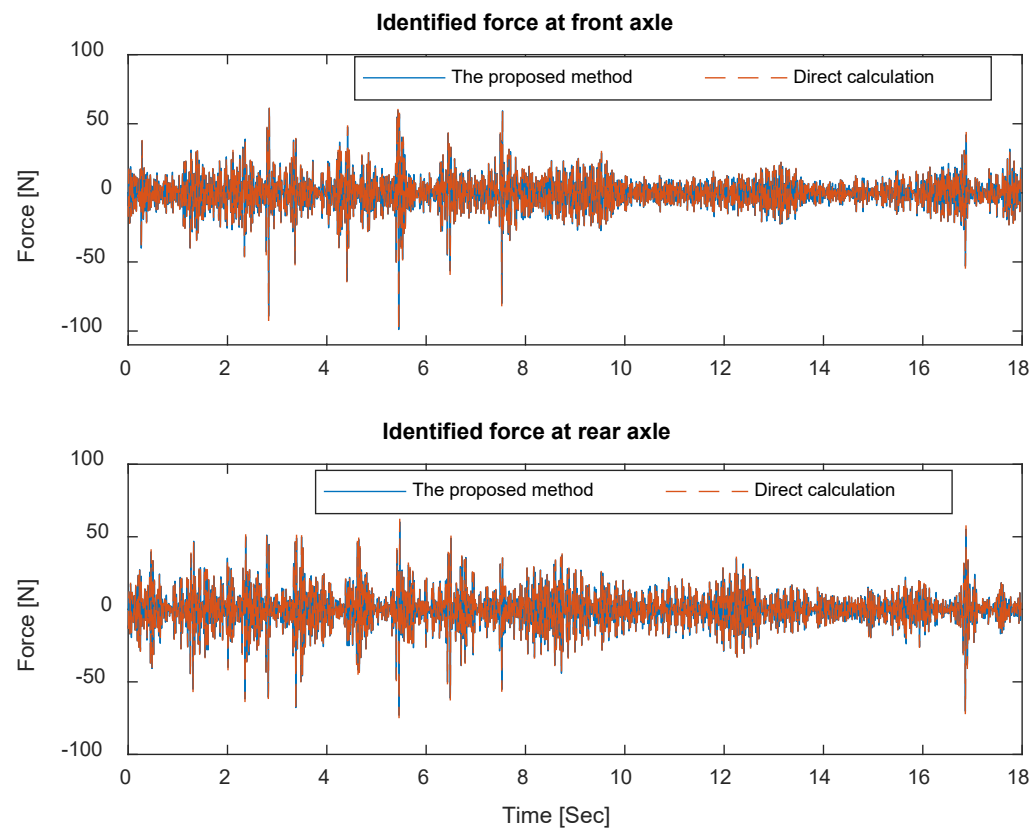

(a) Identified forces 


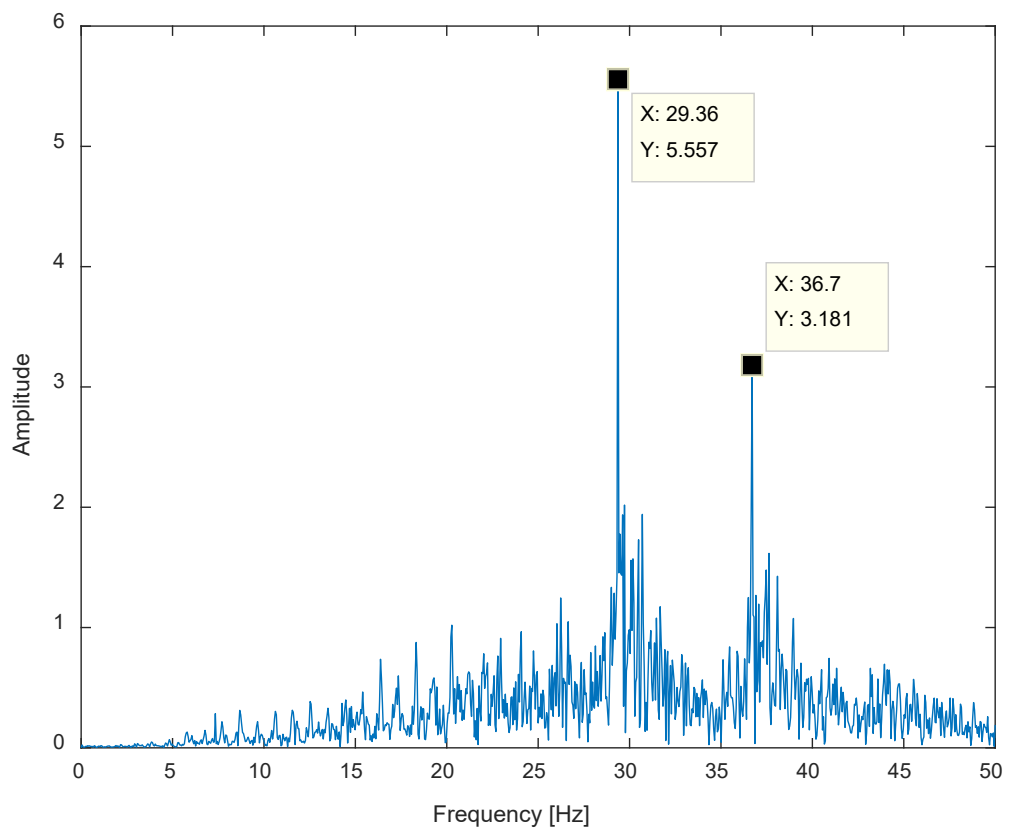

(b) Spectrum of the identified force at front axle

Figure 12 Identified interaction forces and the spectra at two axles

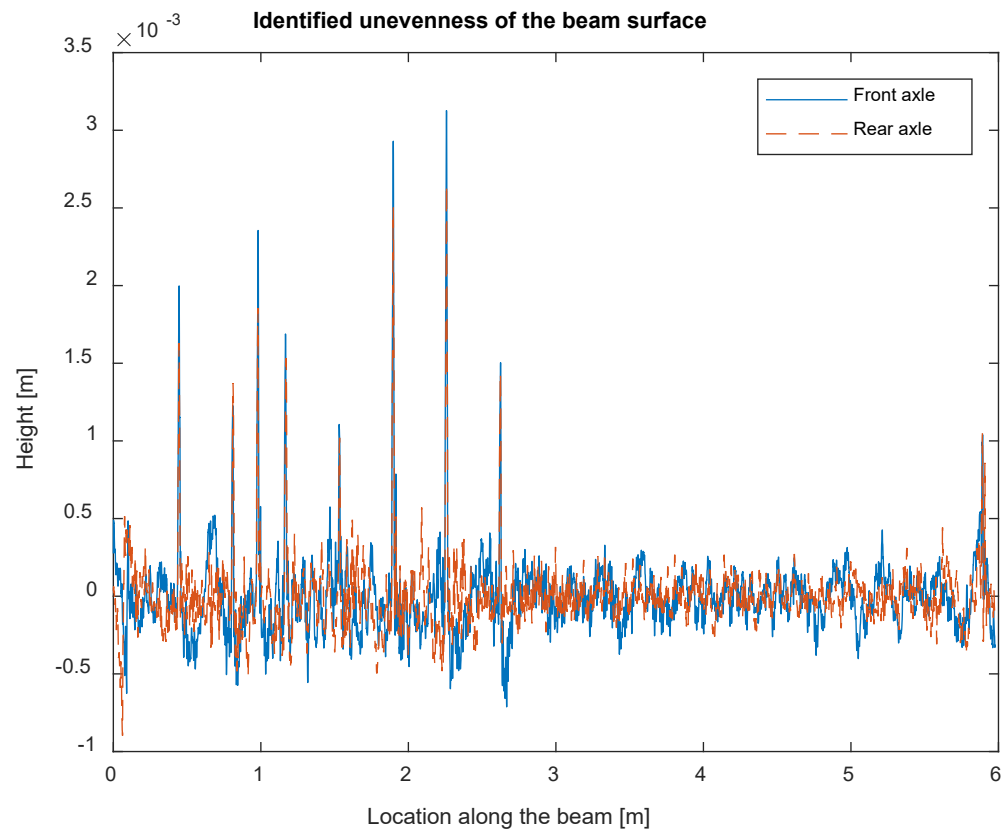

Figure 13 Identified unevenness on the beam surface 


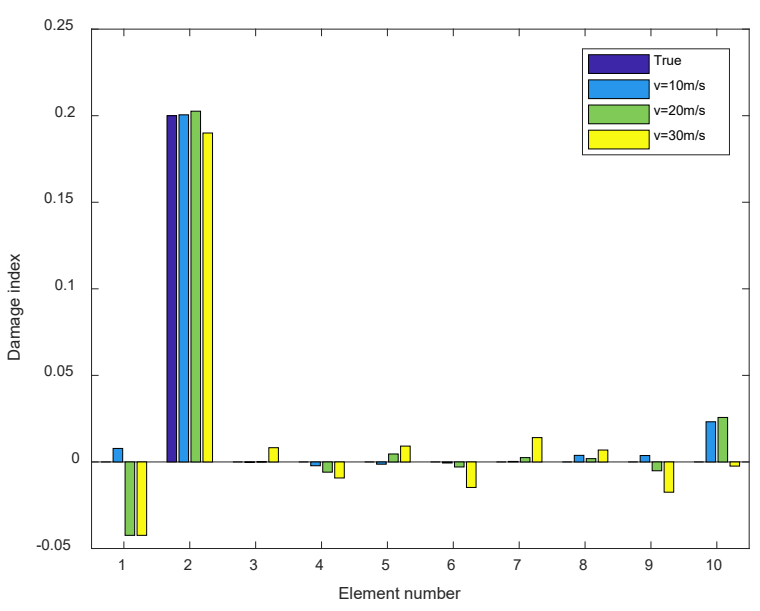

(a) Single damage case

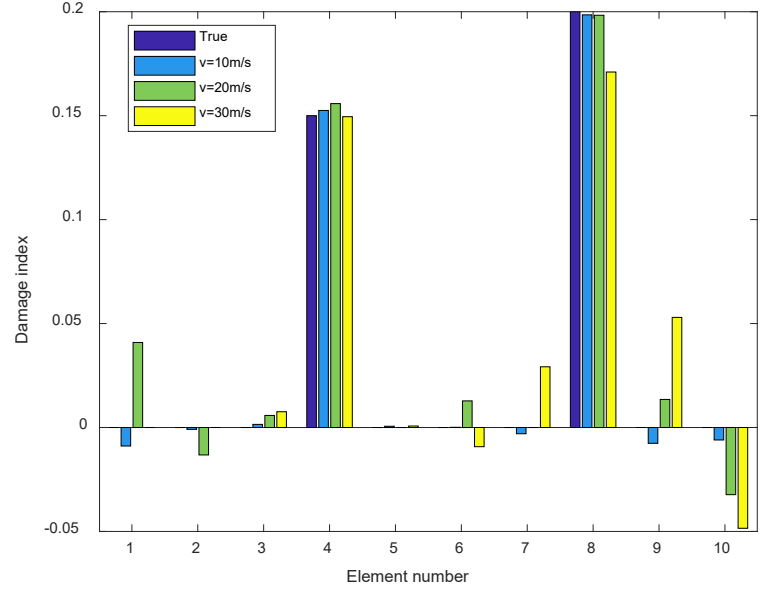

(b) Multiple damage case

Figure 14 Damage detection results considering different vehicle speed when bridge surface is smooth

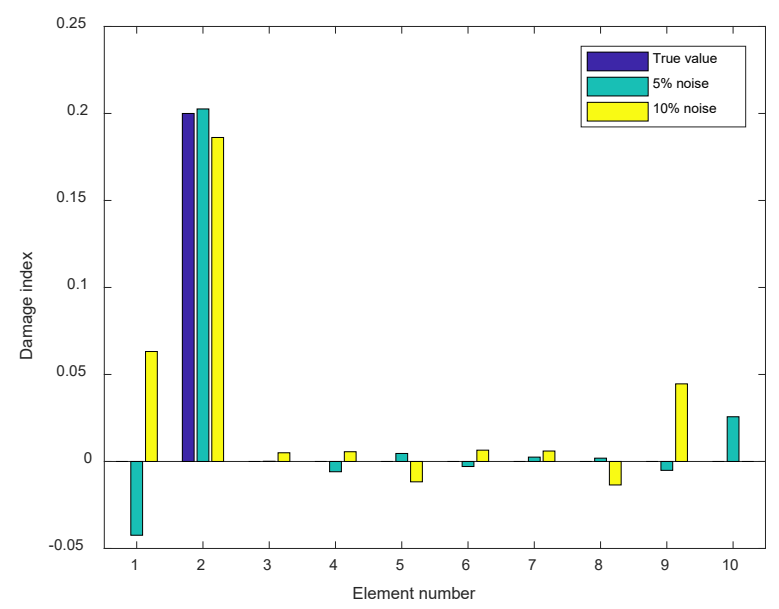

(a) Single damage case

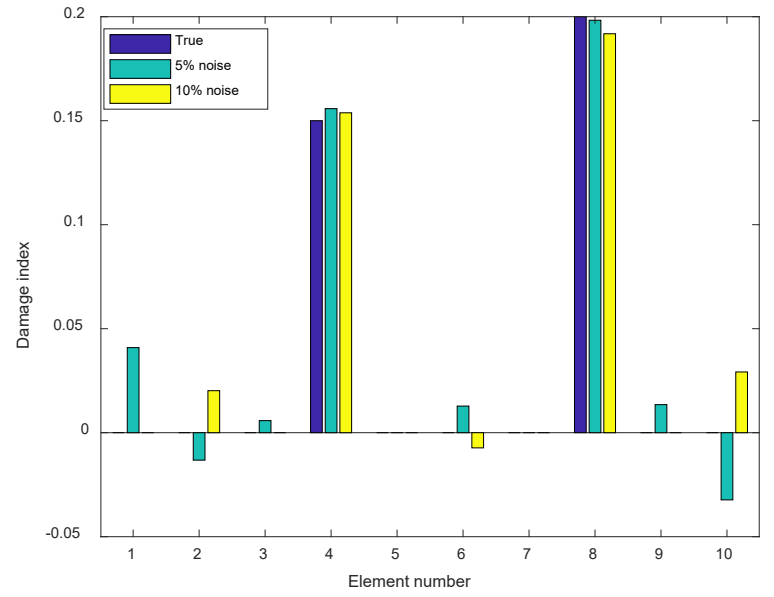

(b) Multiple damage case

Figure 15 Damage detection results considering different measurement noise when bridge surface is smooth 


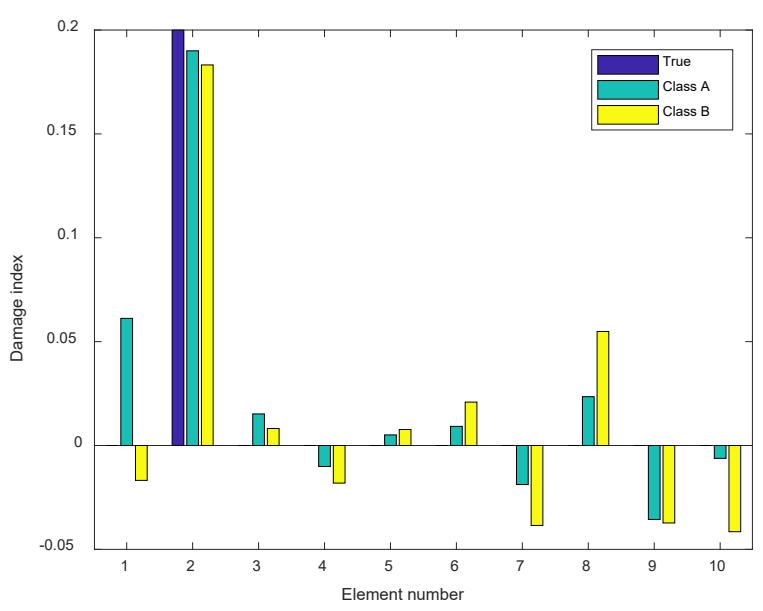

(a) Single damage case

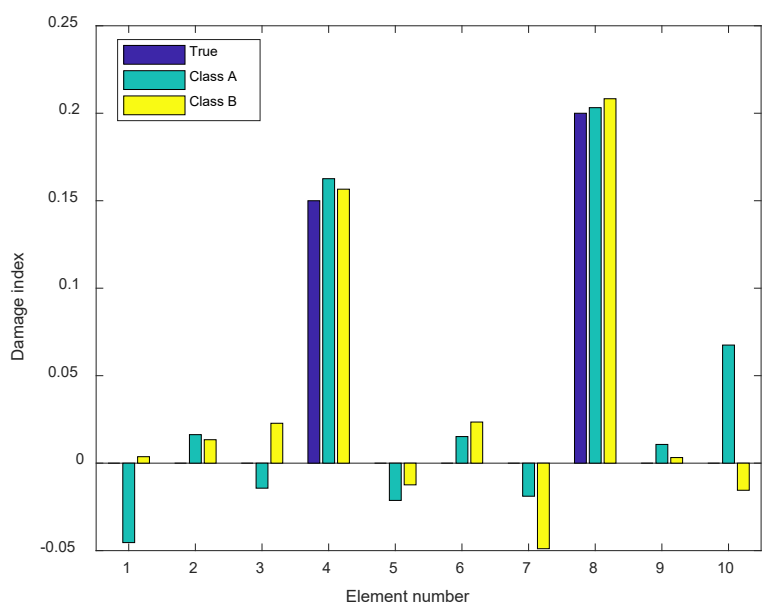

(b) Multiple damage case

Figure 16 Damage detection results when road surface roughness is known

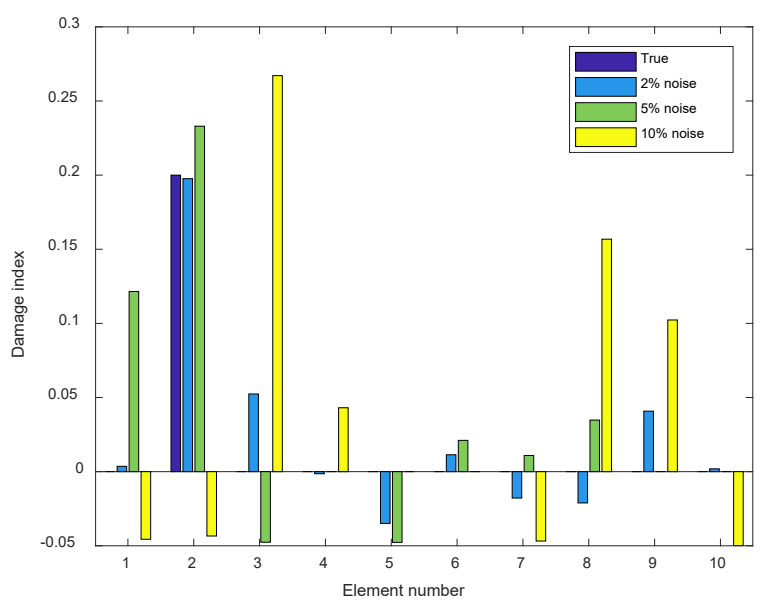

(a) Single damage case

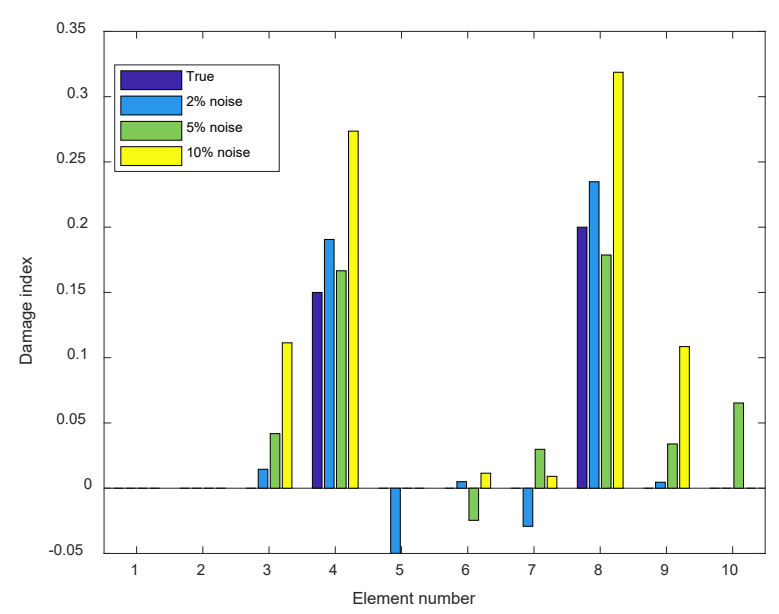

(b) Multiple damage case

Figure 17 Damage detection results when road surface roughness is unknown 